\title{
Weather Conditions Influence on Hyssop Essential Oil Quality
}

\author{
Milica Aćimović ${ }^{1, *(1)}$, Lato Pezo ${ }^{2}{ }^{\oplus}$, Tijana Zeremski ${ }^{1}{ }^{1}$, Biljana Lončar $^{3}{ }^{\circledR}$, Ana Marjanović Jeromela ${ }^{1}$, \\ Jovana Stanković Jeremic ${ }^{4}$, Mirjana Cvetković ${ }^{4}$, Vladimir Sikora ${ }^{1}$ and Maja Ignjatov ${ }^{1}$
}

1 Laboratory for Soil and Agroecology, Department of Vegetable and Alternative Crops, Department of Plant Breeding, Institute of Field and Vegetable Crops Novi Sad, Maksima Gorkog 30, 21000 Novi Sad, Serbia; tijana.zeremski@ifvcns.ns.ac.rs (T.Z.); ana.jeromela@ifvcns.ns.ac.rs (A.M.J.); vladimir.sikora@ifvcns.ns.ac.rs (V.S.); maja.ignjatov@ifvens.ns.ac.rs (M.I.)

2 Engineering Department, Institute of General and Physical Chemistry, University of Belgrade, Studentski Trg 12-16, 11000 Belgrade, Serbia; latopezo@yahoo.co.uk

3 Faculty of Technology, University of Novi Sad, Bulevar Cara Lazara 1, 21000 Novi Sad, Serbia; cbiljana@uns.ac.rs

4 Institute of Chemistry, Technology and Metallurgy, University of Belgrade, Njegoševa 12, 11000 Belgrade, Serbia; jovanas@chem.bg.ac.rs (J.S.J.); miracvet@chem.bg.ac.rs (M.C.)

* Correspondence: milica.acimovic@ifvens.ns.ac.rs

check for updates

Citation: Aćimović, M.; Pezo, L.; Zeremski, T.; Lončar, B.; Marjanović Jeromela, A.; Stanković Jeremic, J.; Cvetković, M.; Sikora, V.; Ignjatov, M. Weather Conditions Influence on Hyssop Essential Oil Quality. Processes 2021, 9, 1152. https:// doi.org/10.3390/pr9071152

Academic Editor:

Jasna Čanadanović-Brunet

Received: 28 May 2021

Accepted: 28 June 2021

Published: 2 July 2021

Publisher's Note: MDPI stays neutral with regard to jurisdictional claims in published maps and institutional affiliations.

Copyright: (c) 2021 by the authors. Licensee MDPI, Basel, Switzerland. This article is an open access article distributed under the terms and conditions of the Creative Commons Attribution (CC BY) license (https:/ / creativecommons.org/licenses/by/ $4.0 /)$.

\begin{abstract}
This paper is a study of the chemical composition of Hyssopus officinalis ssp. officinalis grown during three years (2017-2019) at the Institute of Field and Vegetable Crops Novi Sad (Vojvodina Province, Serbia). Furthermore, comparisons with ISO standards during the years were also investigated, as well as a prediction model of retention indices of compounds from the essential oils. An essential oil obtained by hydrodistillation and analysed by GC-FID and GC-MS was isopinocamphone chemotype. The gathered information about the volatile compounds from $H$. officinalis was used to classify the samples using the unrooted cluster tree. The correlation analysis was applied to investigate the similarity of different samples, according to GC-MS data. The quantitative structureretention relationship (QSRR) was also employed to predict the retention indices of the identified compounds. A total of 74 experimentally obtained retention indices were used to build a prediction model. The coefficient of determination for the training cycle was 0.910 , indicating that this model could be used for the prediction of retention indices for $H$. officinalis essential oil compounds.
\end{abstract}

Keywords: Hyssopus officinalis; essential oil; hydrodistillation; GC-MS; QSRR; artificial neural networks

\section{Introduction}

Hyssopus officinalis L. commonly known as hyssop, is a medicinal, culinary, essential oil-bearing, melliferous and ornamental plant [1,2]. This perennial plant with a strongbranching taproot grows as a subshrub, up to $70 \mathrm{~cm}$ high, with opposite shiny dark green lanceolate or oblong leaves and blue-white or violet flowers arranged in false spikes [3]. During the flowering stage, the aboveground parts (Hyssopi herba) taste slightly bitter and have a strong aromatic flavour, somewhat like a cross between sage and mint [4]. Hyssopus officinalis L. is mostly used for essential oil production. Its essential oil is used in the pharmaceutical and perfume industries and cosmetics as well as in aromatherapy [2-5].

It originates from the Mediterranean region; however, it is well adapted to the northern hemisphere [5]. This plant has two main subspecies in Serbia: ssp. aristatus [syn. ssp. pilifer] (in nature on limestones, dry and sunny slopes, and meadows) and ssp. officinalis (in plantations) [2,6].

The wild-grown subspecies contains linalool as a main compound of the oil, from (35.3 to 51.2)\% [7]. This subspecies does not satisfy the requirements of ISO 9841 standard which does not recognise linalool as the main component of hyssop essential oil and does not have it listed or defined required concentration range. Ref. [8], as well as var. decumbens 
which is also a linalool rich chemotype (51.7\%) [9]. Because of the high content of linalool, it is not used in pharmaceutical or cosmetic industries since linalool can cause allergic reactions on the skin and mucous [10]. Taking this into account, it is very important to know the chemical characteristics of the H. officinalis essential oil for economic use [11].

The quantitative structure retention relationship (QSRR) describes the relation between observed chemical compound structure and its predicted physicochemical or biological properties [12]. The chemical compound structure is explained by molecular descriptors, which encode its data by the symbolic representation of a molecule into a numerical value. Gas Chromatography coupled with Mass Spectrometry (GC-MS) obtains a large number of comparable, reproducible data and exact retention time for large sets of compounds. Lately, various publications have been related to the link of QSRR to GC-MS analysis [13-17]. The mathematical model that represents the relation between the molecular descriptors and the retention time can be established by numerous machine learning algorithms [18], or by using the artificial neural network (ANN), which is used in this study as it has already been proven to be an excellent tool in the published literature [12,19].

This paper reports the results of studies of the chemical composition of Hyssopus officinalis ssp. officinalis essential oil, growing during three growing years (2017-2019) at the Institute of Field and Vegetable Crops Novi Sad (Vojvodina Province, Serbia). Furthermore, the hyssop essential oil characteristic components content were compared with ISO standard requirements which define hyssop essential oil quality. This paper aimed to establish a new QSRR model for predicting the retention times of chemical compounds in H. officinalis essential oil obtained by hydrodistillation and analysed by GC-MS using the genetic algorithm (GA) variable selection method and the artificial neural network (ANN) model.

\section{Materials and Methods}

\subsection{Plant Material}

The study was carried out during three successive growing years: 2016/2017, 2017/ 2018 and 2018/2019 in the experimental field of the Department of Vegetable and Alternative Crops in Bački Petrovac $\left(45^{\circ} 21^{\prime} 36^{\prime \prime}\right.$ N, 19 $35^{\prime} 26^{\prime \prime}$ E), Institute of Field and Vegetable Crops Novi Sad. A small plot with hyssop crop variety "Domaći ljubićasti" was established in sprig 2015 by seeding in rows at an interrow distance of $70 \mathrm{~cm}$. During the vegetation period only hoeing and weeding was performed. Harvest was done in the full flowering stage, manually at the height of $8 \mathrm{~cm}$ above ground during July. After harvest, plant material was dried in a solar dryer at $30^{\circ} \mathrm{C}$. Dried plant material was placed in paper bags for further analysis.

\subsection{Weather Conditions during Growing Years}

Three successive growing years (2016/2017, 2017/2018 and 2018/2019) had significantly different sums of precipitations and average daily temperatures (Figure 1).

Weather conditions in the first growing years (2016/2017): autumn (X-XI) 2016 was characterized by typical temperatures and greater precipitation $(128.0 \mathrm{~mm})$, winter (XII-II) was colder and dry. The beginning of spring (III-V) was hotter than usual and continued with great variability in temperature oscillations in April and average temperatures during May. A typical sum of precipitation was recorded in this period. Summer (VI-VIII) was hotter $\left(24.4^{\circ} \mathrm{C}\right)$ and dryer $(64.7 \mathrm{~mm})$ than average. September was characterized by average values.

Weather conditions in the second growing year (2017/2018): autumn (X-XI) was very favourable with average sums of precipitation and temperatures; winter (XII-II) was hotter with higher precipitation in comparison to the long-term average. Early spring was hotter and more humid, while April and May were hot and dry. Summer was characterized by average temperatures $\left(22.6^{\circ} \mathrm{C}\right)$, but with high precipitation $(220.1 \mathrm{~mm})$. September had average values. 


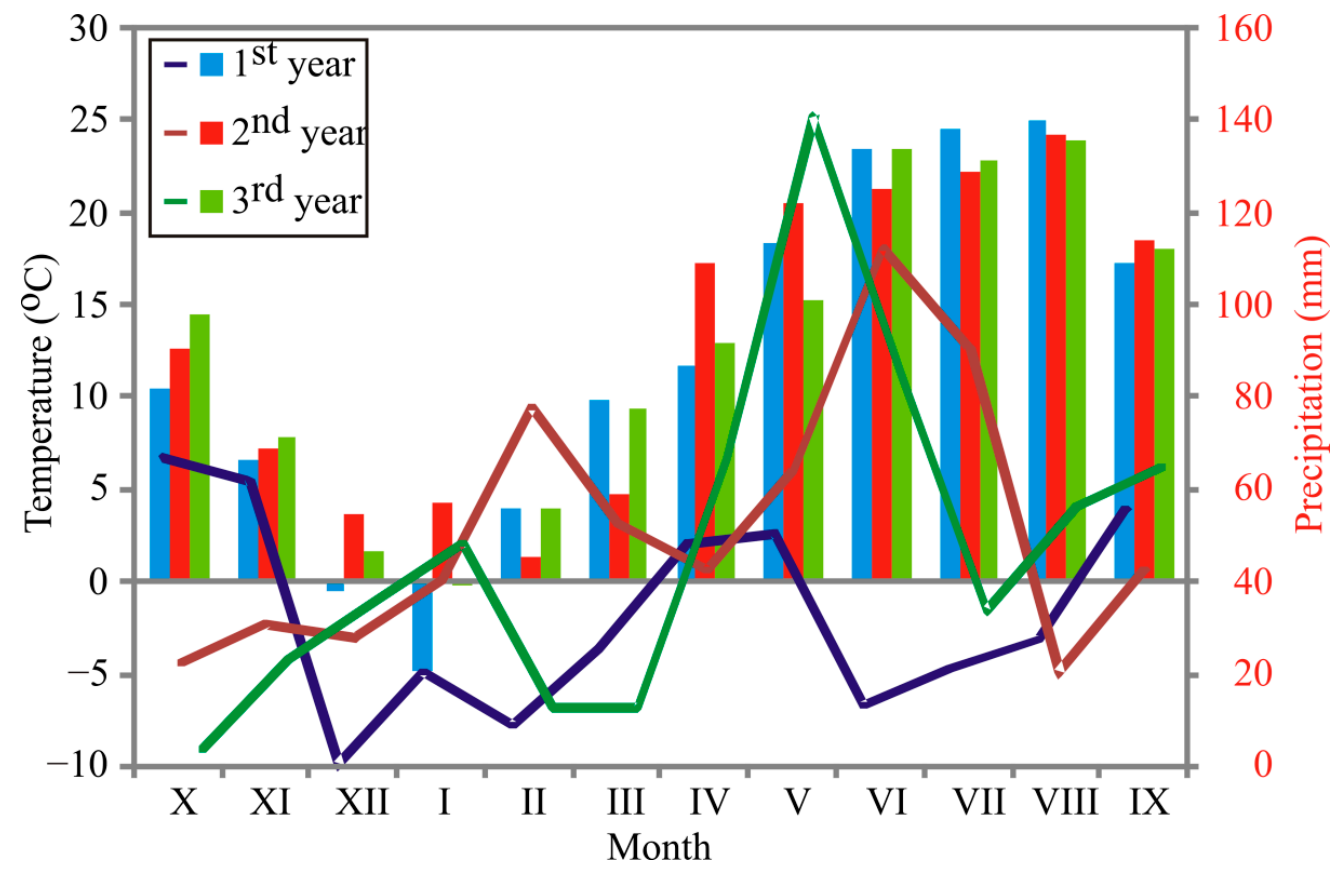

Figure 1. Weather condition during 1st (2016/2017), 2nd (2017/2018) and 3rd (2018/2019) growing seasons. Temperatureswere presented by darker coloured lines, while temperatures were shown by histograms, coloured with pale colours.

Weather conditions in the third growing year (2018/2019): autumn (X-XI) was drier and hotter than usual, winter (XII-II) was hotter with the usual amount of precipitation. The beginning of spring was dry and hot with frost occurrence during March and April, as well as unstable weather with frequent large amounts of precipitation. Summer (VI-VIII) was hot $\left(23.4^{\circ} \mathrm{C}\right)$ and humid $(174 \mathrm{~mm})$. Weather conditions in the third growing year (2018/2019): autumn (X-XI) was drier and hotter than usual; winter (XII-II) was hotter with the usual amount of precipitation. The beginning of spring was dry and hot with frost occurrence during March and April, as well as unstable weather with frequent large amounts of precipitation. Summer (VI-VIII) was hot $\left(23.4^{\circ} \mathrm{C}\right)$ and humid $(174 \mathrm{~mm})$. As the last month of the vegetation period, September was categorized as favourable with hot, dry and sunny weather (important for next growing year).

\subsection{Soil Characteristics}

The soil used for plants growing in this experiment belongs to the chernozem calcareous gleyic type. Basic chemical properties of the soil were determined in samples of the top layer $(0$ to 30$) \mathrm{cm}$. The soil samples were analysed at the Faculty of Agriculture, University of Novi Sad, in accordance with standardized methods adopted in Serbia. Soil $\mathrm{pH}$ value was determined potentiometrically in suspension with $1 \mathrm{M} \mathrm{KCl}$ and suspension with distilled $\mathrm{H}_{2} \mathrm{O}$ (SRPS ISO 10390:2007) [20]. $\mathrm{CaCO}_{3}$ content was determined using the volumetric method (JUS ISO 10693:2005) [21]. The total nitrogen content was determined using the Semimikro-Kjeldahl method modified by Bremner [22]. Available phosphorous and potassium in soil were determined by extraction with ammonium lactate solution (AL) in accordance with the method established by Egner, Riehm and Domingo [23]. Results of agrochemical analyses are shown in Table 1.

Table 1. Basic chemical characteristics of the soil.

\begin{tabular}{|c|c|c|c|c|c|c|}
\hline \multicolumn{2}{|c|}{$\mathrm{pH}$} & \multirow{2}{*}{$\mathrm{CaCO}_{3}(\%)$} & \multirow{2}{*}{ Humus (\%) } & \multirow{2}{*}{$\begin{array}{c}\text { Total } \\
\text { Nitrogen (\%) }\end{array}$} & \multirow{2}{*}{$\begin{array}{l}\text { AL-P }{ }_{2} \mathrm{O}_{5} \\
(\mathrm{mg} / 100 \mathrm{~g})\end{array}$} & \multirow{2}{*}{$\begin{array}{c}\text { AL- } \mathrm{K}_{2} \mathrm{O} \\
(\mathrm{mg} / 100 \mathrm{~g})\end{array}$} \\
\hline In 1M KCl & In $\mathrm{H}_{2} \mathrm{O}$ & & & & & \\
\hline 7.33 & 7.77 & 4.92 & 2.57 & 0.13 & 75.0 & 37.74 \\
\hline
\end{tabular}




\subsection{Isolation Essential Oils and GC-FID GC-MS Analysis}

Essential oils were obtained by hydrodistillation (Clevenger-type apparatus) for $3 \mathrm{~h}$ of the air-dried aerial parts of H. officinalis (42 g 2017, 94 g 2018, 36 g 2019 dry weight, respectively). Essential oils yielded $(0.20,0.74,0.17)$ mass \% calculated to dry plant material from 2017, 2018 and 2019, respectively.

GC-FID and GC-MS analyses were performed according to our previous work [24] with the exception of split ratio which was 10:1 in the present work. The compounds were identified based on a comparison of their linear retention index relative to $\mathrm{C}_{8}-\mathrm{C}_{32} n$-alkanes and mass spectra with MS data library (Adams4 and NIST17). The relative mass percentage concentrations were computed from GC-FID peak areas.

\subsection{QSRR Analysis}

The molecular structure dataset was presented in short ASCII files (with extension. smi, the simplified molecular-input line). The molecular data were collected from the PubChem database (https: / / pubchem.ncbi.nlm.nih.gov/, accessed on 30 January 2021). The calculation of the specified molecular descriptors for each chemical compound [25] was performed using free molecular descriptor software PaDel-descriptor [26]. A huge amount of data was obtained for each chemical compound, and it was necessary to use a genetic algorithm (GA) $[27,28]$, and the calculation was performed using Heuristic Lab (https:/ / dev.heuristiclab.com/trac.fcgi/, accessed on 30 January 2021), to select the most relevant molecular descriptors for retention time prediction. In this study, GA was used to determine the most appropriate molecular descriptors to develop a reliable model for predicting the retention time of the compounds found in H. officinalis essential oil. The correlation between the descriptors was examined, and collinear descriptors were detected using factor analysis.

\subsection{Artificial Neural Network (ANN)}

A multi-layer perceptron model (MLP), which is explained by the input, hidden and output layers, was used in this paper, considering that it is well known as capable of approximating nonlinear functions [29]. Broyden-Fletcher-Goldfarb-Shanno (BFGS) algorithm was used for ANN modelling. The input and output data were normalized to improve the behaviour of the ANN. The experimental database was randomly divided into training (60\%), testing $(20 \%)$ and validation parts $(20 \%)$ for ANN modelling. ANN results, including the weight values, depending on the initial assumptions of parameters necessary for ANN construction and fitting [30,31]. A series of different topologies were used, in which the number of hidden neurons varied from 5 to 20 and the training process of the network was run 100,000 times with random initial values of weights and biases. The optimization process was performed based on validation error minimization. Statistical investigation of the data has been performed mainly by the Statistica 10 software [32].

\subsection{Global Sensitivity Analysis}

Yoon's interpretation method was used to determine the relative influence (RIN) of molecular descriptors on retention time [33]. This method was applied based on the weight coefficients of the developed ANN.

$$
\operatorname{RIN}_{i j}(\%)=\frac{\sum_{k=0}^{n}\left(w_{i k} \cdot w_{k j}\right)}{\sum_{i=0}^{m}\left|\sum_{k=0}^{n}\left(w_{i k} \cdot w_{k j}\right)\right|} \cdot 100 \%
$$

where: $w$-weight coefficient in ANN model, $i$-input variable, $j$-output variable, $k-$ hidden neuron, $n-$ number of hidden neurons, $m$-number of inputs. 


\subsection{Cluster Analysis}

The cluster analysis (CA) was employed to evaluate the variations of the EO's compounds content of $H$. officinalis samples gathered in different sites and/or taken from literature reports. The phylogenetic tree diagram for obtained samples was plotted using the R software 4.0.3 (64-bit version). The R package "ape" (Analysis of Phylogenetics and Evolution) was utilized for calculation ("unrooted" tree cluster analysis was plotted). The distance matrix was calculated using the Euclidean method, using the "complete" method.

\subsection{Linear Regression Model}

A linear regression model was developed to predict the H. officinalis essential oil active compound content according to temperature and precipitation data, and the appropriate regression coefficients were calculated.

\subsection{Correlation Analysis}

The correlation analysis was performed using the R software, to present the similarities between different samples graphically.

\subsection{Statistical Analyses}

The collected data were processed statistically using the software package STATISTICA 10.0 [32].

\section{Results and Discussion}

\subsection{GC-MS Analysis of the Hyssop Essential Oil}

The main compounds in hyssop essential oil during all three years were isopinocamphone (38.8 to 43.8$) \%$, pinocamphone (18.3 to 22.3 ) \% and $\beta$-pinene (6.3 to 12.0$) \%$, followed by $\beta$-phellandrene (in average $3.4 \%$ ), pinocarvone (3.2\%), germacrene $\mathrm{D}(2.2 \%)$, myrtenol and elemol (both in an average of $1.7 \%)$, bicyclogermacrene (1.6\%), myrcene $(1.4 \%), \beta-$ caryophyllene and one NI compound (both in average 1.3\%), sabinene and limonene (both in average $1.2 \%$ ), Table 2 .

On isopinocamphone as the most abundant component $(41.2 \%$ on average for three years), the influence of temperatures was negative (regression coefficient was -26.218), while the impact of precipitation was positive (1.336) on the accumulation. This effect explains the reduced accumulation in the 2 nd year of the study $(38.8 \%)$ when the mean daily temperatures were higher and the total rainfall during the growing year compared to the other years of the research. On the other hand, the influence of increased temperatures was positive on the accumulation of pinocamphone (regression coefficient was 20.860) and $\beta$-pinene (7.006), while the impact of precipitation was negative $(-1.065$ and -0.693 , respectively).

According to the ISO 9841 Standard [8], the content of pinocamphone, isopinocamphone, $\beta$-caryophyllene, germacrene $\mathrm{D}$, elemol and spathulenol satisfied requirements during all three years, while $\alpha$-pinene, $\beta$-pinene, sabinene, limonene, $\beta$-bourbonene and allo-aromadendrene was slightly exceeded in some years. Myrtenyl methyl ether was not detected in this investigation (Table 3). Therefore, the high quality of H. officinalis essential oil indicates that there is interest in growing this species in plantations in Serbia. 
Table 2. H. officinalis essential oil composition during three growing years; temperature and precipitation regression coefficients in the linear model for compound content prediction.

\begin{tabular}{|c|c|c|c|c|c|c|c|c|c|}
\hline \multirow{2}{*}{ No } & \multirow{2}{*}{ Compound } & \multirow{2}{*}{$R I_{\text {pred }}$} & \multirow{2}{*}{$R I$} & \multicolumn{3}{|c|}{ Compound Content (\%) } & \multirow[b]{2}{*}{ Average \pm SD } & \multicolumn{2}{|c|}{$\begin{array}{l}\text { Regression } \\
\text { Coefficients }\end{array}$} \\
\hline & & & & $\begin{array}{c}\text { 1st Year } \\
\text { [34] }\end{array}$ & $\begin{array}{c}\text { 2nd Year } \\
\text { [35] }\end{array}$ & $\begin{array}{c}\text { 3rd Year } \\
{[2]}\end{array}$ & & $\begin{array}{l}\text { Temp. } \\
\text { Coeff. }\end{array}$ & $\begin{array}{l}\text { Prec. } \\
\text { Coeff. }\end{array}$ \\
\hline 1 & $\alpha$-Thujene (MT) & 992.07 & 925 & 0.3 & 0.1 & 0.1 & $0.2 \pm 0.1$ & 0.198 & -0.022 \\
\hline 2 & $\alpha$-Pinene (MT) & 956.96 & 932 & 0.7 & 0.3 & 0.3 & $0.4 \pm 0.2$ & 0.394 & -0.044 \\
\hline 3 & Camphene (MT) & 976.15 & 947 & 0.1 & 0.1 & 0.1 & $0.1 \pm 0.0$ & 0.000 & 0.000 \\
\hline 4 & Thuja-2,4(10)-diene (MT) & 1022.53 & 952 & $\operatorname{tr}$ & - & - & $\operatorname{tr}$ & 0.000 & 0.000 \\
\hline 5 & Sabinene (MT) & 1006.67 & 972 & 1.7 & 1.1 & 0.9 & $1.2 \pm 0.4$ & 1.724 & -0.130 \\
\hline 6 & $\beta$-Pinene (MT) & 971.80 & 975 & 12.0 & 6.6 & 6.3 & $8.3 \pm 3.2$ & 7.006 & -0.693 \\
\hline 7 & Myrcene (MT) & 904.58 & 990 & 1.5 & 1.6 & 1.0 & $1.4 \pm 0.3$ & 3.312 & -0.179 \\
\hline 8 & $\mathrm{NI}$ & & 992 & - & - & 0.1 & $\operatorname{tr}$ & & \\
\hline 9 & $\alpha$-Terpinene (MT) & 982.03 & 1016 & 0.1 & 0.2 & 0.1 & $0.1 \pm 0.1$ & 0.469 & -0.021 \\
\hline 10 & p-Cymene (MT) & 995.14 & 1023 & 0.1 & 0.1 & 0.2 & $0.1 \pm 0.1$ & -0.570 & 0.032 \\
\hline 11 & Limonene (MT) & 953.43 & 1026 & - & - & 3.5 & $1.2 \pm 2.0$ & -19.944 & 1.114 \\
\hline 12 & $\beta$-Phellandrene (MT) & 1009.23 & 1028 & 4.1 & 5.9 & 0.3 & $3.4 \pm 2.9$ & 30.050 & -1.578 \\
\hline 13 & 1,8-Cineole (OMT) & 1183.38 & 1030 & 0.3 & - & - & $0.1 \pm 0.2$ & 0.296 & -0.033 \\
\hline 14 & cis- $\beta$-Ocimene (MT) & 882.25 & 1036 & $\operatorname{tr}$ & 0.2 & 0.1 & $0.1 \pm 0.1$ & 0.370 & -0.010 \\
\hline 15 & trans- $\beta$-Ocimene (MT) & 882.25 & 1046 & 0.1 & 0.6 & 0.5 & $0.4 \pm 0.3$ & 0.074 & 0.024 \\
\hline 16 & $\gamma$-Terpinene (MT) & 971.35 & 1057 & 0.2 & 0.4 & 0.3 & $0.3 \pm 0.1$ & 0.371 & -0.010 \\
\hline 17 & cis-Sabinene hydrate (MT) & 1025.19 & 1066 & 0.1 & 0.4 & 0.4 & $0.3 \pm 0.2$ & -0.298 & 0.033 \\
\hline 18 & Terpinolene (MT) & 918.64 & 1088 & - & 0.1 & 0.1 & $0.1 \pm 0.1$ & -0.099 & 0.011 \\
\hline 19 & Linalool (OMT) & 1047.20 & 1100 & 0.4 & 0.5 & 0.6 & $0.5 \pm 0.1$ & -0.669 & 0.043 \\
\hline 20 & cis-Thujone (OMT) & 1138.15 & 1106 & 0.1 & 0.2 & 0.2 & $0.2 \pm 0.1$ & -0.096 & 0.011 \\
\hline 21 & trans-Thujone (OMT) & 1138.15 & 1116 & 0.1 & 0.2 & 0.1 & $0.1 \pm 0.1$ & 0.469 & -0.021 \\
\hline 22 & NI & & 1121 & - & 0.1 & 0.1 & $0.1 \pm 0.1$ & & \\
\hline 23 & Nopinone (O) & 1044.29 & 1136 & 0.1 & $\operatorname{Tr}$ & 0.1 & $0.1 \pm 0.0$ & -0.470 & 0.021 \\
\hline 24 & trans-Pinocarveol (OMT) & 1094.78 & 1140 & 0.1 & 0.3 & 0.5 & $0.3 \pm 0.2$ & -1.340 & 0.086 \\
\hline 25 & NI & & 1157 & 1.8 & 1.0 & 1.1 & $1.3 \pm 0.44$ & & \\
\hline 26 & Pinocamphone (OMT) & 1095.79 & 1158 & 20.5 & 22.3 & 18.3 & $20.4 \pm 2.0$ & 20.860 & -1.065 \\
\hline 27 & Pinocarvone (OMT) & 1083.90 & 1162 & 2.8 & 0.7 & 6.1 & $3.2 \pm 2.7$ & -28.715 & 1.486 \\
\hline 28 & Borneol (OMT) & 1082.91 & 1164 & - & $\operatorname{Tr}$ & - & $\operatorname{tr}$ & 0.000 & 0.000 \\
\hline 29 & Isopinocamphone (OMT) & 1095.79 & 1174 & 41.1 & 38.8 & 43.8 & $41.2 \pm 2.5$ & -26.218 & 1.336 \\
\hline 30 & Terpinen-4-ol (OMT) & 1091.37 & 1179 & 0.7 & 0.9 & - & $0.5 \pm 0.5$ & 4.899 & -0.263 \\
\hline 31 & Cryptone $(\mathrm{O})$ & 1075.55 & 1184 & - & $\operatorname{Tr}$ & - & $\operatorname{tr}$ & 0.000 & 0.000 \\
\hline 32 & NI & & 1185 & - & - & 0.1 & $\operatorname{tr}$ & & \\
\hline 33 & $\alpha$-Terpineol (OMT) & 1100.45 & 1190 & 0.2 & 0.2 & 0.2 & $0.2 \pm 0.0$ & 0.000 & 0.000 \\
\hline 34 & Myrtenol (OMT) & 1099.28 & 1195 & 1.1 & 2.0 & 2.0 & $1.7 \pm 0.5$ & -0.894 & 0.100 \\
\hline 35 & Methyl chavicol (PP) & 1146.03 & 1197 & - & - & 0.3 & $0.1 \pm 0.2$ & -1.712 & 0.096 \\
\hline 36 & $\begin{array}{c}\text { trans-2-hydroxy-Pinocamphone } \\
\text { (OMT) }\end{array}$ & 1095.79 & 1246 & 0.1 & - & 0.1 & $0.1 \pm 0.1$ & -0.470 & 0.021 \\
\hline 37 & Methyl myrtenate (OMT) & 1280.48 & 1296 & 0.1 & 0.1 & 0.2 & $0.1 \pm 0.1$ & -0.570 & 0.032 \\
\hline 38 & Myrtenyl acetate (OMT) & 1385.53 & 1325 & 0.1 & - & 0.2 & $0.1 \pm 0.1$ & -1.041 & 0.053 \\
\hline 39 & $\alpha$-Copaene (ST) & 1510.08 & 1375 & $\operatorname{tr}$ & $\operatorname{Tr}$ & - & $\operatorname{tr}$ & 0.000 & 0.000 \\
\hline 40 & $\delta$-elemene (ST) & 1450.99 & 1334 & - & 0.1 & 0.6 & $0.2 \pm 0.3$ & -2.954 & 0.171 \\
\hline 41 & $\beta$-Bourbonene (ST) & 1503.87 & 1384 & 0.6 & 0.9 & 0.5 & $0.7 \pm 0.2$ & 1.970 & -0.093 \\
\hline 42 & $\beta$-Elemene (ST) & 1428.91 & 1389 & - & $\operatorname{tr}$ & 0.1 & $\operatorname{tr}$ & -0.570 & 0.032 \\
\hline 43 & Methyl eugenol (PP) & 1385.60 & 1405 & - & 0.1 & 0.2 & $0.1 \pm 0.1$ & -0.670 & 0.043 \\
\hline 44 & $\alpha$-Gurjunene (ST) & 1513.34 & 1409 & 0.2 & 0.4 & 0.2 & $0.3 \pm 0.1$ & 0.936 & -0.041 \\
\hline 45 & $\beta$-Caryophyllene (ST) & 1444.73 & 1419 & 1.5 & 1.4 & 1.1 & $1.3 \pm 0.2$ & 1.797 & -0.106 \\
\hline 46 & $\beta$-Copaene (ST) & 1516.98 & 1429 & $\operatorname{tr}$ & 0.1 & 0.1 & $0.1 \pm 0.0$ & -0.099 & 0.011 \\
\hline 47 & 6,9-Guaiadiene (ST) & 1532.04 & 1444 & $\operatorname{tr}$ & 0.1 & 0.1 & $0.1 \pm 0.0$ & -0.099 & 0.011 \\
\hline 48 & $\alpha$-Humulene (ST) & 1384.87 & 1453 & 0.2 & 0.2 & 0.2 & $0.2 \pm 0.0$ & 0.000 & 0.000 \\
\hline 49 & trans- $\beta$-Farnesene (ST) & 1397.11 & 1456 & - & - & 0.1 & $\operatorname{tr}$ & -0.570 & 0.032 \\
\hline 50 & allo-Aromadendrene (ST) & 1509.53 & 1459 & - & 1.4 & - & $0.5 \pm 0.8$ & 6.550 & -0.288 \\
\hline 51 & 9-epi- $\beta$-Caryophyllene (ST) & 1444.73 & 1461 & 0.7 & - & 0.7 & $0.5 \pm 0.4$ & -3.299 & 0.145 \\
\hline 52 & cis-Muurola-4(14),5-diene (ST) & 1531.12 & 1466 & $\operatorname{tr}$ & - & - & $\operatorname{tr}$ & 0.000 & 0.000 \\
\hline 53 & Germacrene (DST) & 1483.09 & 1482 & 1.7 & 3.0 & 2.0 & $2.2 \pm 0.6$ & 4.381 & -0.172 \\
\hline 54 & $\beta$-Selinene (ST) & 1468.06 & 1487 & - & 0.1 & - & $\operatorname{tr}$ & 0.469 & -0.021 \\
\hline 55 & $\mathrm{NI}$ & & 1489 & $\operatorname{tr}$ & - & 0.1 & $\operatorname{tr}$ & & \\
\hline 56 & Bicyclogermacrene (ST) & 1434.42 & 1497 & 1.3 & 2.5 & 0.9 & $1.6 \pm 0.8$ & 7.877 & -0.373 \\
\hline 57 & $\gamma$-Cadinene (ST) & 1506.17 & 1515 & $\operatorname{tr}$ & 0.1 & - & $\operatorname{tr}$ & 0.469 & -0.021 \\
\hline 58 & $\mathrm{NI}$ & & 1521 & - & - & 0.1 & $\operatorname{tr}$ & & \\
\hline 59 & $\delta$-Cadinene (ST) & 1500.65 & 1523 & 0.2 & 0.1 & 0.1 & $0.1 \pm 0.1$ & 0.099 & -0.011 \\
\hline 60 & Elemol (OST) & 1566.08 & 1550 & 1.0 & 1.7 & 2.5 & $1.7 \pm 0.8$ & -5.262 & 0.333 \\
\hline
\end{tabular}


Table 2. Cont.

\begin{tabular}{|c|c|c|c|c|c|c|c|c|c|}
\hline \multirow{2}{*}{ No } & \multirow{2}{*}{ Compound } & \multirow{2}{*}{$R I_{\text {pred }}$} & \multirow{2}{*}{$R I$} & \multicolumn{3}{|c|}{ Compound Content ( $\%)$} & \multirow[b]{2}{*}{ Average \pm SD } & \multicolumn{2}{|c|}{$\begin{array}{l}\text { Regression } \\
\text { Coefficients }\end{array}$} \\
\hline & & & & $\begin{array}{c}\text { 1st Year } \\
{[34]}\end{array}$ & $\begin{array}{c}\text { 2nd Year } \\
\text { [35] }\end{array}$ & $\begin{array}{c}\text { 3rd Year } \\
{[2]}\end{array}$ & & $\begin{array}{l}\text { Temp. } \\
\text { Coeff. }\end{array}$ & $\begin{array}{l}\text { Prec. } \\
\text { Coeff. }\end{array}$ \\
\hline 61 & Spathulenol (OST) & 1603.77 & 1577 & 0.4 & 0.6 & 0.7 & $0.6 \pm 0.2$ & -0.769 & 0.054 \\
\hline 62 & Caryophyllene oxide (OST) & 1587.07 & 1583 & 0.2 & 0.4 & 0.5 & $0.4 \pm 0.2$ & -0.769 & 0.054 \\
\hline 63 & NI & & 1600 & - & 0.2 & 0.2 & $0.1 \pm 0.1$ & & \\
\hline 64 & Veridiflorol (OST) & 1600.87 & 1601 & 0.1 & - & - & $\operatorname{tr}$ & 0.099 & -0.011 \\
\hline 65 & 10-epi- $\gamma$-Eudesmol (OST) & 1620.23 & 1619 & $\operatorname{tr}$ & - & - & $\operatorname{tr}$ & 0.000 & 0.000 \\
\hline 66 & $\gamma$-Eudesmol (OST) & 1598.78 & 1632 & 0.2 & 0.3 & 0.1 & $0.2 \pm 0.1$ & 1.034 & -0.052 \\
\hline 67 & NI & & 1636 & - & 0.1 & 0.1 & $0.1 \pm 0.1$ & & \\
\hline 68 & epi- $\alpha$-Cadinol $(=\tau$-cadinol) (OST) & 1635.68 & 1641 & 0.1 & 0.1 & - & $0.1 \pm 0.1$ & 0.567 & -0.032 \\
\hline 69 & NI & & 1639 & - & - & 0.1 & $\operatorname{tr}$ & & \\
\hline 70 & $\beta$-Eudesmol (OST) & 1599.400 & 1650 & 0.2 & 0.2 & 0.1 & $0.2 \pm 0.1$ & 0.567 & -0.032 \\
\hline 71 & $\alpha$-Eudesmol (OST) & 1592.25 & 1654 & 0.2 & 0.3 & 0.2 & $0.2 \pm 0.1$ & 0.469 & -0.021 \\
\hline 72 & NI & & 1662 & $\operatorname{tr}$ & 0.1 & - & $\operatorname{tr}$ & & \\
\hline 73 & NI & & 1669 & $\operatorname{tr}$ & 0.1 & - & $\operatorname{tr}$ & & \\
\hline 74 & NI & & 1845 & - & - & 0.1 & $\operatorname{tr}$ & & \\
\hline & MT: Monoterpene hydrocarbons & & & 21.0 & 17.7 & 14.2 & & & \\
\hline & OMT: Oxygenated monoterpenes & & & 67.7 & 66.2 & 72.3 & & & \\
\hline & ST: Sesquiterpene hydrocarbons & & & 6.4 & 10.4 & 6.7 & & & \\
\hline & OST: Oxygenated sesquiterpenes & & & 2.4 & 3.6 & 4.1 & & & \\
\hline & PP: Phenylpropanoids & & & - & 0.1 & 0.5 & & & \\
\hline & O: Other & & & 0.1 & - & 0.1 & & & \\
\hline & NI: Not Identified & & & 1.8 & 1.6 & 2.1 & & & \\
\hline & Total identified & & & 99.4 & 99.6 & 100.0 & & & \\
\hline
\end{tabular}

Compound content for 1st, 2nd and 3rd year were taken from the literature: [34], [35] and [2], respectively. Temp. coeff.-temperature coefficient in linear model of chemical compounds content in essential oil; Prec. coeff.-precipitations coefficient in linear model of chemical compounds content in essential oil; $R I$-retention indices (relative to C8-C36 n-alkanes on the HP-5MSI column); $R I_{\text {pred. }}-$ Retention Index predicted by QSRR model.

Table 3. Comparison results of main H. officinalis essential oil components from studies [34], [35] and [2], and ISO 9841 standard.

\begin{tabular}{cccccc}
\hline & \multicolumn{3}{c}{ This Study } & \multicolumn{2}{c}{ ISO 9841 Standard } \\
\cline { 2 - 6 } Component & $\begin{array}{c}\text { 1st Year } \\
{[34]}\end{array}$ & $\begin{array}{c}\text { 2nd Year } \\
\text { [35] }\end{array}$ & $\begin{array}{c}\text { 3rd Year } \\
\text { [2] }\end{array}$ & Min & Max \\
\hline$\alpha$-Pinene & 0.7 & 0.3 & 0.3 & 0.4 & 1.5 \\
$\beta$-Pinene & 12.0 & 6.6 & 6.3 & 7.0 & 20.0 \\
Sabinene & 1.7 & 1.1 & 0.9 & 1.0 & 3.5 \\
Limonene & 0.0 & 0.0 & 3.5 & 0.6 & 4.0 \\
Myrtenyl methyl ether & 0.0 & 0.0 & 0.0 & 0.9 & 3.0 \\
Pinocamphone * & 20.5 & 22.3 & 18.3 & 8.0 & 25.0 \\
Isopinocamphone * & 41.1 & 38.8 & 43.8 & 25.0 & 45.0 \\
$\beta$-Bourbonene & 0.6 & 0.9 & 0.5 & 0.8 & 2.6 \\
$\beta$-Caryophyllene * & 1.5 & 1.4 & 1.1 & 1.0 & 3.0 \\
allo-aromadendrene & 0.0 & 1.4 & 0.0 & 1.0 & 3.0 \\
Germacrene D * & 1.7 & 3.0 & 2.0 & 1.2 & 4.5 \\
Elemol * & 1.0 & 1.7 & 2.5 & 0.2 & 2.5 \\
Spathulenol * & 0.4 & 0.6 & 0.7 & 0.1 & 1.5
\end{tabular}

* Satisfies requirements during all three years. Compound content for 1st, 2nd and 3rd year were taken from the literature: [34], [35] and [2], respectively.

\subsection{Climatic Factors and Their Influence on Hyssop Essential Oil Chemical Composition}

The chemical composition variations of $H$. officinalis oils can be related to many factors. Still, the most important is intraspecific diversity, as a consequence of adaptive evolution on diverse habitats (geographical and climatic) and technological influences (the stages of development and harvesting time, the part used, irrigation) [36,37]. However, our previous studies were dealing with chemical composition and biological activities [2,34,35]. It is known that biological activity depends on the chemical composition of essential oil $[38,39]$. 
During the three-year experimental period (2012-2014), the vegetation year significantly affected essential oil yield but not the essential oil composition [40]. Also, during three years of cultivation of hyssop in Finland, the content of pinocamphone varied between 18.0 and $19.7 \%$, isopinocamphone between 29.2 and $32.6 \%$, germacrene $\mathrm{D}$ between 6.7 and $10.2 \%$, $\beta$-pinene between 5.2 and $6.6 \%$ and pinocarvone between 5.3 and $5.7 \%$ [41]. In addition, investigations conducted in Ukraine for three years showed significant variations in the content of pinocamphone (from 35.49 to $53.73 \%$ ) and isopinocamphone (4.66 to $44.43) \%$ depending on plant age [42].

A strong negative correlation was observed between the amount of precipitation and the accumulation level of volatile organic compounds in the flowering shoots: rain during the development of flowers seems to have a negative effect on the yield [40]. Trans-pinocamphone was predominant in the oil during the vegetative stage, however, its content decreased with plant growth, while the content of pinocamphone increased [43], gradually from the pre-blooming stage $(47.94 \%)$ through the full-blooming $(48.22 \%)$ to the post-blooming stage (51.42\%) [11]. This phenomenon indicated that choosing a suitable harvesting stage to achieve the highest essential oil quality was crucial [11]. It is known that saturated bicyclic monoterpene ketones pinocamphone and isopinocamphone pathway rise from $\beta$-pinene via allyc alcohol trans-pinocarveol by subsequent oxidation and two stereochemical alternatives for reduction of the conjugated double bond [44].

This plant is a typical xerophyte and is well adapted to drought [11]. Furthermore, it is also cold and frost tolerant [45]. Draught stress increases the number of essential oil compounds (from 27 in non-stress plants to 42 in intense drought stress conditions). In addition, with water deficit, the content of cis-pinocamphone is decreased [46]. In semiarid conditions, with 300-400 mm annual rainfall, the content of isopinocamphone was $19.8 \%$ and pinocamphone was $17.2 \%$, while in irrigated crops contents of both compounds increased to $23.6 \%$ and $18.5 \%$, respectively [47]. However, severe drought citrulline could increase the yield of isopinocamphone, so it could be a novel metabolite agent used for improving qualitative parameters in hyssop cropping systems [5]. In addition, salt stress influences the relative content of components in essential oil. Pinocarvone content was significantly decreased with increasing $\mathrm{NaCl}$ levels, while isopinocamphone being the least affected [48].

\subsection{Unrooted Cluster Tree Analysis}

Table 4 is shown 70 accessions of $H$. officinalis essential oil from literature, with 25 most abundant compounds (on average more than $0.5 \%$ ). According to the unrooted cluster tree (Figure 2), it could be said that there are several chemotypes. Still, they mainly contain a mixture of pinocamphone + isopinocamphone $+\beta$-pinene in different proportions but also appear as a particular chemotype. 
Table 4. Chemical composition of different hyssop accessions according to literature.

\begin{tabular}{|c|c|c|c|c|c|c|c|c|c|c|c|c|c|c|c|c|c|c|c|c|c|c|c|c|c|c|c|}
\hline No & 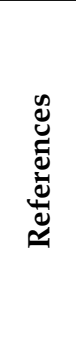 & 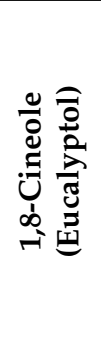 & 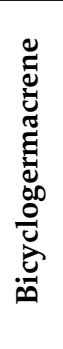 & 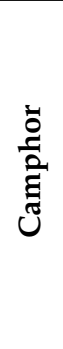 & 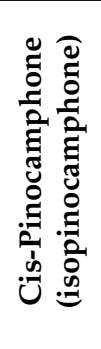 & 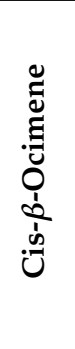 & $\begin{array}{l}\overline{\overrightarrow{0}} \\
\overline{\mathrm{d}} \\
\overline{\mid r}\end{array}$ & 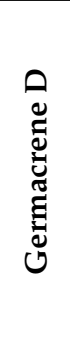 & 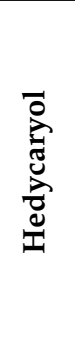 & 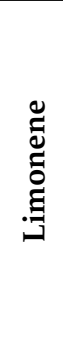 & 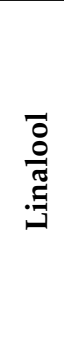 & 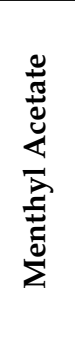 & 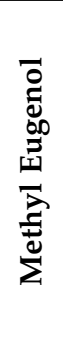 & 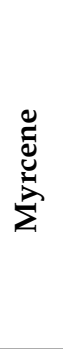 & $\underset{\sum}{\stackrel{D}{Z}}$ & 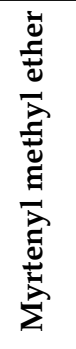 & 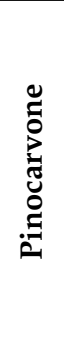 & 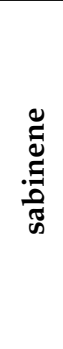 & 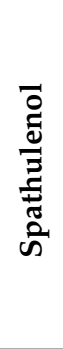 & 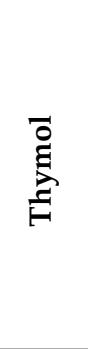 & 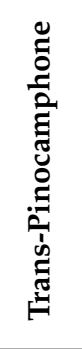 & 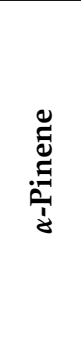 & $\begin{array}{l}\tilde{E} \\
\tilde{0} \\
\tilde{0} \\
0 \\
\vdots \\
0 \\
0 \\
0 \\
0\end{array}$ & 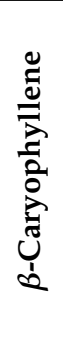 & 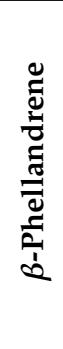 & 苞 & 䒕 \\
\hline 2 & [50] & 0.5 & 0.0 & 0.0 & 43.3 & 0.0 & 0.0 & 0.9 & 0.0 & 1.6 & 0.0 & 0.0 & 0.0 & 0.5 & 0.0 & 0.0 & 0.0 & 0.0 & 0.0 & 0.0 & 16.8 & 1.0 & 1.2 & 1.0 & 0.0 & 16.3 & 83.1 \\
\hline 3 & [51] & 0.2 & 1.8 & 0.0 & 49.9 & 0.1 & 0.0 & 1.9 & 0.0 & 1.5 & 0.5 & 0.2 & 0.1 & 1.9 & 1.5 & 1.6 & 0.2 & 1.6 & 0.4 & 0.0 & 5.9 & 0.9 & 1.3 & 1.0 & 4.8 & 13.5 & 90.8 \\
\hline 4 & [52] & 7.6 & 2.0 & 0.0 & 1.9 & 0.0 & 0.0 & 0.0 & 0.0 & 1.4 & 0.1 & 0.0 & 0.0 & 3.9 & 0.6 & 0.0 & 0.0 & 3.0 & 0.3 & 0.0 & 51.0 & 1.0 & 0.2 & 1.0 & 0.0 & 12.4 & 86.4 \\
\hline 5 & [52] & 1.9 & 3.3 & 0.0 & 21.9 & 0.0 & 5.4 & 0.1 & 0.0 & 1.0 & 0.8 & 0.0 & 0.4 & 1.7 & 4.7 & 0.0 & 0.0 & 2.2 & 1.0 & 0.0 & 28.8 & 0.7 & 0.8 & 0.9 & 0.0 & 9.8 & 85.4 \\
\hline 6 & [11] & 0.3 & 0.0 & 0.0 & 57.3 & 0.0 & 0.6 & 0.0 & 0.0 & 0.6 & 0.8 & 1.0 & 0.0 & 0.5 & 0.0 & 0.0 & 6.5 & 0.9 & 0.6 & 0.6 & 2.6 & 0.3 & 0.0 & 0.4 & 0.0 & 7.2 & 80.0 \\
\hline 7 & [53] & 36.4 & 0.8 & 0.0 & 15.3 & 0.0 & 0.0 & 1.7 & 0.0 & 0.0 & 0.3 & 0.0 & 0.0 & 2.0 & 0.0 & 0.0 & 0.0 & 2.9 & 0.4 & 0.0 & 6.4 & 2.6 & 0.6 & 1.1 & 0.0 & 19.6 & 89.9 \\
\hline 8 & [54] & 0.2 & 3.4 & 0.0 & 58.5 & 0.6 & 0.0 & 2.6 & 0.0 & 0.0 & 0.0 & 0.0 & 0.0 & 0.0 & 0.0 & 2.3 & 0.0 & 1.3 & 0.0 & 0.0 & 0.0 & 0.4 & 0.7 & 0.8 & 4.8 & 8.5 & 84.1 \\
\hline 9 & [54] & 0.3 & 2.2 & 0.0 & 66.9 & 0.2 & 0.0 & 2.9 & 0.0 & 0.0 & 0.0 & 0.0 & 0.0 & 0.0 & 0.8 & 3.2 & 0.0 & 1.0 & 0.0 & 0.0 & 0.0 & 0.2 & 0.5 & 0.7 & 3.6 & 4.2 & 86.5 \\
\hline 10 & [54] & 0.2 & 0.6 & 0.0 & 63.3 & 0.0 & 0.0 & 1.8 & 0.0 & 0.0 & 0.0 & 0.0 & 0.0 & 0 . & 0.3 & 2.6 & 0.0 & 1.5 & 0.0 & 0.0 & 0. & 0.3 & 0.6 & 0.8 & 6.8 & 7.4 & 86.2 \\
\hline 13 & [40] & 0.0 & 2.5 & 0.0 & 53.5 & 0.0 & 1.4 & 1.6 & 0.0 & 0.0 & 0.0 & 0.0 & 0.0 & 1.2 & 0.7 & 0.0 & 4.3 & 1.2 & 0.0 & 0.0 & 6.4 & 0.0 & 0.0 & 0.0 & 2.8 & 8.1 & 83.5 \\
\hline 14 & [40] & 0.0 & 1.4 & 0.0 & 61.3 & 0.0 & 0.8 & 0.6 & 0.0 & 0.0 & 0.0 & 0.0 & 0.0 & 1.1 & 1.7 & 0.0 & 3.7 & 0.1 & 0.0 & 0.0 & 1.4 & 0.0 & 0.0 & 0.0 & 4.6 & 8.8 & 85.3 \\
\hline 15 & [40] & 0.0 & 0.9 & 0.0 & 33.2 & 0.0 & 0.7 & 0.7 & 0.0 & 0.0 & 0.0 & 0.0 & 0.0 & 1.5 & 2.4 & 0.0 & 4.1 & 1.5 & 0.0 & 0.0 & 31.1 & 0.0 & 0.0 & 0.0 & 4.2 & 13.0 & 93.0 \\
\hline 16 & [55] & 1.2 & 0.6 & 3.5 & 0.0 & 0.0 & 0.0 & 1.3 & 0.0 & 3.0 & 1.0 & 0.0 & 0.0 & 0.0 & 0.0 & 0.0 & 0.0 & 0.0 & 3.0 & 19.0 & 0.0 & 0.3 & 0.4 & 1.6 & 0.0 & 0.0 & 34.8 \\
\hline 17 & [56] & 0.0 & 0.0 & 0.0 & 44.7 & 0.0 & 5.6 & 1.6 & 0.0 & 0.0 & 1.1 & 0.0 & 0.4 & 0.8 & 2.8 & 0.0 & 0.0 & 5.2 & 2.8 & 0.0 & 14.1 & 0.0 & 0.0 & 1.3 & 2.4 & 0.0 & 82.8 \\
\hline 18 & [57] & 0.0 & 1.8 & 0.0 & 26.9 & 0.0 & 8.0 & 1.7 & 0.0 & 1.6 & 1.1 & 0.0 & 0.9 & 0.0 & 1.7 & 0.0 & 0.0 & 0.0 & 0.3 & 0.0 & 16.0 & 0.0 & 0.0 & 2.3 & 2.4 & 20.4 & 85.0 \\
\hline 19 & [58] & 38.6 & 0.0 & 0.0 & 7.0 & 11.6 & 0.0 & 1.4 & 0.0 & 0.0 & 0.0 & 0.0 & 0.0 & 0.9 & 0.0 & 0.0 & 0.7 & 2.3 & 0.0 & 0.0 & 0.1 & 2.3 & 0.3 & 0.9 & 0.0 & 9.2 & 75.2 \\
\hline 20 & [58] & 35.9 & 0.0 & 0.0 & 7.8 & 14.4 & 0.0 & 1.3 & 0.0 & 0.0 & 0.0 & 0.0 & 0.0 & 1.0 & 0.0 & 0.0 & 0.6 & 2.4 & 0.0 & 0.0 & $\operatorname{tr}$ & 2.0 & 0.2 & 1.0 & 0.0 & 7.9 & 74.6 \\
\hline 21 & [59] & 4.2 & 1.6 & 0.0 & 0.0 & 0.0 & 0.5 & 3.5 & 0.0 & 0.0 & 0.8 & 0.0 & 0.5 & 0.0 & 1.9 & 0.0 & 0.0 & 0.0 & 0.4 & 19.7 & 15.3 & 1.4 & 0.6 & 4.3 & 0.0 & 6.6 & 61.2 \\
\hline 22 & [4] & 1.1 & 0.0 & 0.5 & 39.4 & 1.1 & 0.7 & 0.6 & 0.0 & 0.4 & 0.6 & 0.0 & 0.3 & 0.3 & 1.7 & 0.0 & 6.1 & 0.6 & 0.1 & 0.7 & 14.1 & 1.2 & 1.5 & 0.4 & 0.0 & 1.7 & 73.0 \\
\hline 23 & [60] & 2.1 & 0.0 & 1.5 & 72.8 & 0.3 & 0.0 & 0.0 & 0.0 & 0.0 & 0.7 & 0.0 & 0.0 & 1.0 & 0.0 & 0.0 & 0.9 & 0.8 & 1.7 & 0.0 & 0.7 & 0.3 & 0.0 & 0.0 & 1.3 & 3.7 & 87.8 \\
\hline 24 & [47] & 0.0 & 0.5 & 0.0 & 21.7 & 0.0 & 1.1 & 3.8 & 0.0 & 1.8 & 1.2 & 0.0 & 0.5 & 0.0 & 1.4 & 3.1 & 0.0 & 2.7 & 0.3 & 0.0 & 17.9 & 1.7 & 4.0 & 3.7 & 3.1 & 18.9 & 87.4 \\
\hline 25 & [61] & 0.3 & 0.0 & 6.8 & 0.0 & 0.0 & 0.5 & 3.4 & 0.0 & 0.0 & 0.6 & 74.1 & 0.0 & 0.0 & 0.0 & 0.0 & 0.0 & 0.0 & 2.1 & 0.0 & 0.0 & 0.0 & 1.5 & 2.1 & 0.0 & 0.0 & 91.2 \\
\hline 26 & [43] & 0.0 & 1.8 & 0.0 & 39.2 & $\operatorname{tr}$ & 4.3 & 4.4 & 0.0 & 0.0 & 0.6 & 0.0 & 0.0 & 1.6 & 2.5 & 0.0 & 0.7 & 1.3 & 0.5 & $\operatorname{tr}$ & 28.3 & 0.2 & 0.6 & 1.7 & 2.9 & 7.2 & 97.6 \\
\hline
\end{tabular}


Table 4. Cont.

\begin{tabular}{|c|c|c|c|c|c|c|c|c|c|c|c|c|c|c|c|c|c|c|c|c|c|c|c|c|c|c|c|}
\hline No & 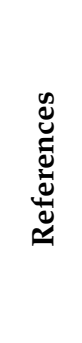 & 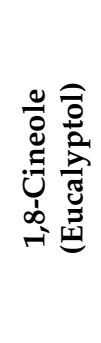 & 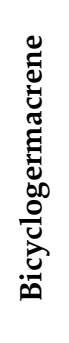 & 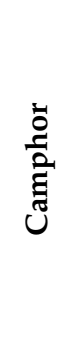 & 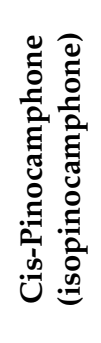 & 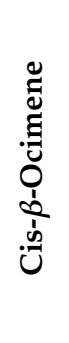 & $\frac{\overline{0}}{\stackrel{\Xi}{0}}$ & 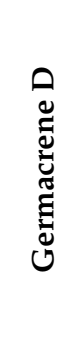 & 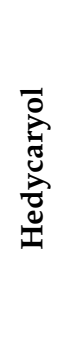 & 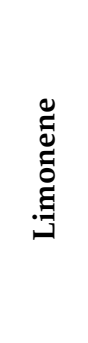 & $\begin{array}{l}\overline{8} \\
\stackrel{0}{\Xi}\end{array}$ & 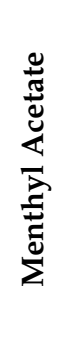 & 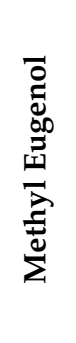 & 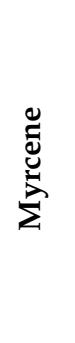 & 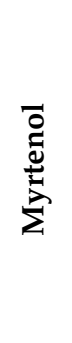 & 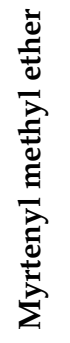 & 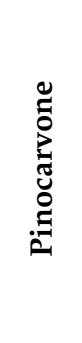 & 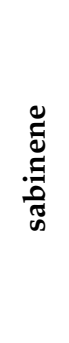 & 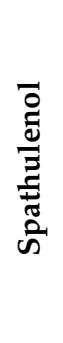 & 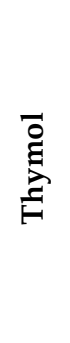 & 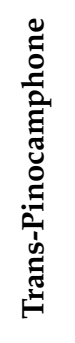 & 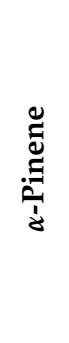 & 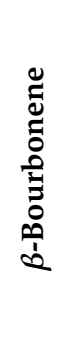 & 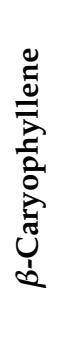 & 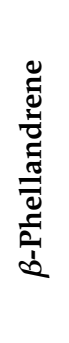 & 苞 & 胥 \\
\hline 28 & [9] & 12.3 & 0.0 & 0.0 & 1.4 & 0.0 & 0.0 & 0.0 & 0.0 & 5.1 & 51.7 & 0.0 & 0.0 & 1.3 & 0.0 & 0.0 & 0.0 & 0.8 & 0.0 & 0.0 & 1.0 & 2.2 & 1.0 & 2.4 & 0.0 & 3.0 & 82.2 \\
\hline 29 & [9] & 0.0 & 0.0 & 0.0 & 43.3 & 0.0 & 1.7 & 0.0 & 0.0 & 12.2 & 0.0 & 0.0 & 4.0 & 2.1 & 0.0 & 0.0 & 0.0 & 1.5 & 0.0 & 0.0 & 4.4 & 0.6 & 1.4 & 1.5 & 0.0 & 11.1 & 83.8 \\
\hline 30 & [63] & 0.0 & 2.1 & 0.0 & 15.5 & 0.0 & 0.0 & 3.8 & 4.2 & 0.8 & 2.5 & 0.0 & 0.0 & 2.0 & 2.6 & 0.0 & 28.1 & 2.5 & 2.2 & 0.0 & $\operatorname{tr}$ & 0.8 & 1.1 & 2.9 & 3.6 & 8.7 & 83.4 \\
\hline 31 & [63] & 0.0 & 2.5 & 0.0 & 15.9 & 0.0 & 0.0 & 3.7 & 4.3 & 1.0 & 2 & 0. & 0.0 & 1.9 & & 0.0 & 26.9 & & 2.3 & 0.0 & 0.1 & 0.5 & 1.1 & 2.3 & 3.1 & 7.0 & 80.0 \\
\hline 32 & [63] & 0.0 & 2.4 & 0.0 & 14.2 & $\operatorname{tr}$ & 0.0 & 4.1 & 4.1 & 1.1 & 2 & 0. & 0.0 & 1. & 3 & 0.0 & 24.5 & & 2.0 & 0.0 & & 0.5 & 1.5 & 3.1 & 3.4 & 10.2 & 79.3 \\
\hline 33 & [63] & 0.0 & 2.4 & 0.0 & 11.5 & 0.0 & 0.0 & 5.5 & 4.8 & 0.5 & 1.3 & 0.0 & 0.0 & 1.0 & 2.7 & 0.0 & 21.1 & 0.9 & 3.0 & 0.0 & 0.0 & 0.5 & 1.2 & 2.2 & 2.5 & 11.4 & 72.5 \\
\hline 34 & [63] & 0.0 & 2.4 & 0.0 & 33.6 & 1.2 & 0.0 & 0.0 & 4.0 & 0.5 & 0.9 & 0.0 & 0.0 & 1.2 & 2.5 & 0.0 & 9.0 & 0.9 & 2.2 & 0.0 & 0.3 & 0.5 & 1.2 & 1.1 & 1.7 & 7.2 & 70.4 \\
\hline 35 & [63] & 0.0 & 3.3 & 0.0 & 16.8 & 0.3 & 0.0 & 0.0 & 9.1 & 0.7 & 1.0 & 0.0 & 0.0 & 1.0 & 2.4 & 0.0 & 13.6 & 0.8 & 2.1 & 0.0 & 0.2 & 0.4 & 1.5 & 4.0 & 1.5 & 8.6 & 67.3 \\
\hline 36 & [64] & 0.0 & 0.0 & 13.1 & 1.2 & 0.1 & 0.0 & 3.1 & 8.1 & 0.7 & 0.0 & 0.0 & 0.0 & 0.0 & 2.3 & 1.3 & 0.0 & 1.0 & 1.1 & 0.0 & 34.7 & 1.2 & 0.0 & 3.3 & 3.3 & 7.3 & 82.0 \\
\hline 39 & [65] & 0.0 & 2.3 & 0.0 & 33.7 & 0.1 & 3.2 & 3.4 & 0.0 & 0.9 & 1.4 & 0.0 & 0.3 & 1.4 & 1.7 & 3.5 & 9.2 & 1.3 & 1.3 & 0.0 & 16.9 & 0.3 & 0.0 & 1.4 & 3.3 & 7.2 & 92.5 \\
\hline 40 & [35] & 0.0 & 2.2 & 0.0 & 45.1 & 0.0 & 1.9 & 2.8 & 0.0 & 0.0 & 0.9 & 0.0 & 0.1 & 1.5 & 1.5 & 0.0 & 2.7 & 1.1 & 0.5 & 0.0 & 16.4 & 0.3 & 0.7 & 1.6 & 5.6 & 6.4 & 91.3 \\
\hline 41 & [35] & 0.3 & 1.0 & 0.0 & 16.1 & 0.2 & 0.0 & 1.6 & 0.6 & 0.0 & 0.3 & 0.0 & $\operatorname{tr}$ & 0.8 & 2.5 & 0.0 & 0.5 & 1.1 & 0.4 & 0.0 & 58.3 & 0.4 & 1.1 & 0.8 & 0.0 & 8.4 & 94.4 \\
\hline 42 & [35] & 0.0 & 2.5 & 0.0 & 38.8 & 0.2 & 1.7 & 3.0 & 0.0 & 0.0 & 0.5 & 0.0 & 0.1 & 1.6 & 2.0 & 0.0 & 0.7 & 1.1 & 0.6 & 0.0 & 22.3 & 0.3 & 0.9 & 1.4 & 5.9 & 6.6 & 90.2 \\
\hline 43 & [34] & 0.3 & 1.3 & 0.0 & 41.1 & $\operatorname{tr}$ & 1.0 & 1.7 & 0.0 & 0.0 & 0.4 & 0.0 & 0.0 & 1.5 & 1.1 & 0.0 & 2.8 & 1.7 & 0.4 & 0.0 & 20.5 & 0.7 & 0.6 & 1.5 & 4.1 & 12.0 & 92.7 \\
\hline 44 & [66] & 0.0 & 0.0 & 0.0 & 9.7 & 0.1 & 0.0 & 0.7 & 0.0 & 5.6 & 0.0 & 0.0 & 0.2 & 0.0 & 0.7 & 0.0 & 0.0 & 1.3 & 0.0 & 0.0 & 49.1 & 1.8 & 0.0 & 0.3 & 4.2 & 18.4 & 91.9 \\
\hline 45 & [67] & 7.2 & 0.6 & 0.2 & 5.3 & 0.0 & 0.0 & 1.0 & 0.0 & 0.5 & 0.1 & 0.0 & 0.0 & 2.5 & 0.0 & 0.0 & 36.3 & 1.0 & 0.9 & 0.0 & 19.6 & 0.8 & 0.1 & 0.4 & 0.0 & 10.6 & 87.1 \\
\hline 46 & [41] & 0.0 & 4.5 & 0.0 & 30.4 & 0.7 & 0.0 & 8.6 & 4.8 & 0.5 & 0.1 & 0.0 & 0.0 & 1.0 & 1.8 & 1.2 & 5.5 & 0.9 & 1.1 & 0.0 & 19.1 & 0.4 & 0.0 & 2.4 & 2.6 & 6.0 & 91.7 \\
\hline 47 & [41] & 0.0 & 5.8 & & 16.7 & 0.1 & 0.0 & 11.9 & 3.0 & 0.5 & 0.3 & 0.0 & 0.0 & 1.1 & 2.2 & 0.9 & 25.7 & 1.1 & 1.2 & 0.0 & 1.9 & 0.6 & 0.0 & 2.5 & 3.3 & 12.9 & 91.9 \\
\hline 48 & [41] & 0.0 & 5.0 & 0.0 & 1.5 & 1.3 & 0.0 & 9.5 & 5.8 & 0.4 & 0.6 & 0.0 & 0.0 & 0.7 & 2.4 & 0.4 & 1.7 & 0.9 & 1.2 & 0.0 & 47.0 & 0.3 & 0.0 & 2.4 & 0.5 & 8.3 & 89.8 \\
\hline 49 & [46] & 0.0 & 3.0 & 0.0 & 35.9 & 0.0 & 7.2 & 1.8 & 0.0 & 0.1 & 0.6 & 0.0 & 0.0 & 1.1 & 2.4 & 0.0 & 0.0 & 1.1 & 0.3 & 0.0 & 22.1 & 0.4 & 0.7 & 1.4 & 1.5 & 9.3 & 88.7 \\
\hline 50 & [68] & 6.4 & 0.0 & 0.0 & 30.7 & 0.0 & 0.0 & 0.2 & 0.0 & 0.0 & 0.3 & 0.0 & 0.0 & 1.0 & 2.6 & 0.0 & 32.1 & 2.2 & 0.1 & 0.0 & 0.0 & 0.7 & 0.0 & 0.0 & 2.5 & 15.3 & 94.0 \\
\hline 51 & [69] & 0.0 & 1.6 & 0.3 & 3.2 & 0.0 & 0.0 & 5.1 & 0.0 & 0.0 & 0.2 & 0.0 & 0.0 & 3.5 & 0.0 & 0.0 & 0.0 & 0.4 & 2.3 & 0.0 & 34.0 & 2.1 & 0.0 & 5.6 & 0.0 & 10.5 & 68.8 \\
\hline 52 & [69] & 0.0 & 1.4 & 5.3 & 29.0 & 0.0 & 0.0 & 3.3 & 0.0 & 0.0 & 7.9 & 0.0 & 0.0 & 1.6 & 0.0 & 0.0 & 0.0 & 0.4 & 1.4 & 0.0 & 18.5 & 2.1 & 0.0 & 2.4 & 0.0 & 10.8 & 84.1 \\
\hline
\end{tabular}


Table 4. Cont.

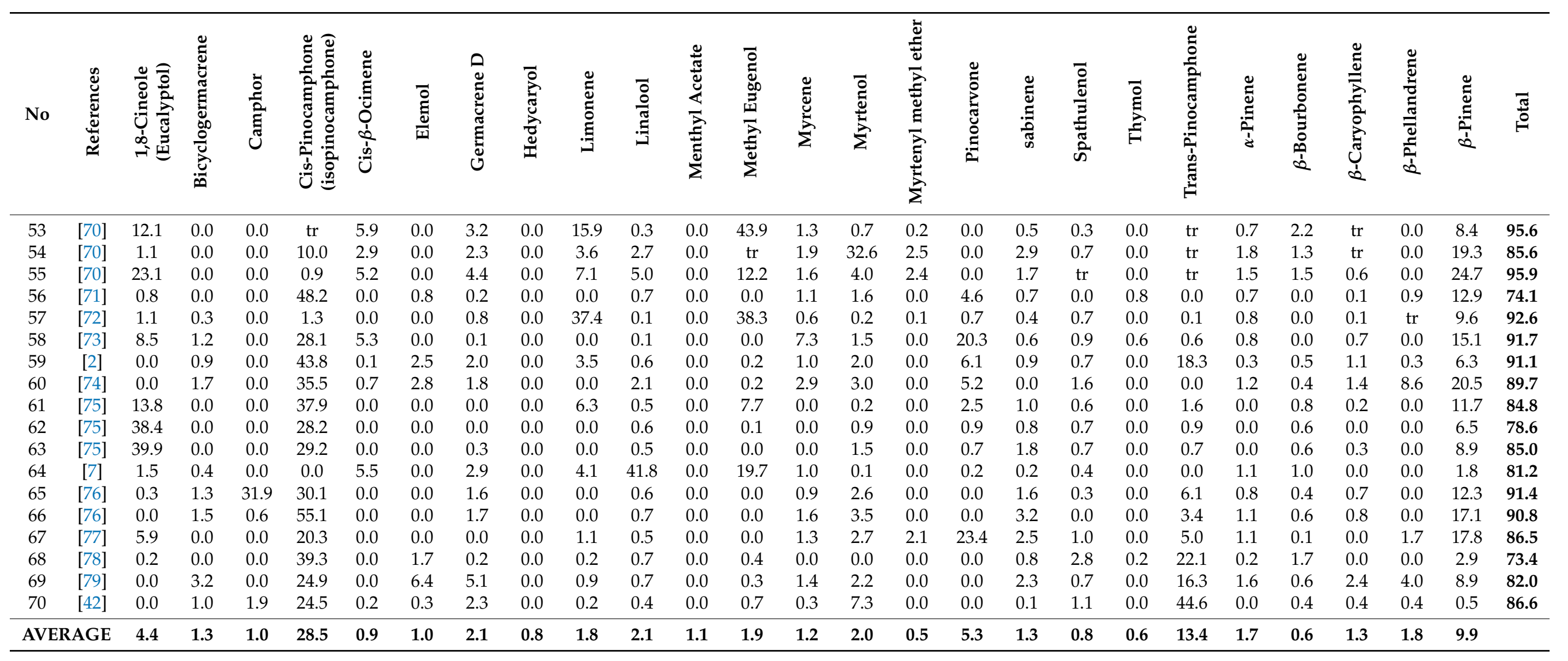




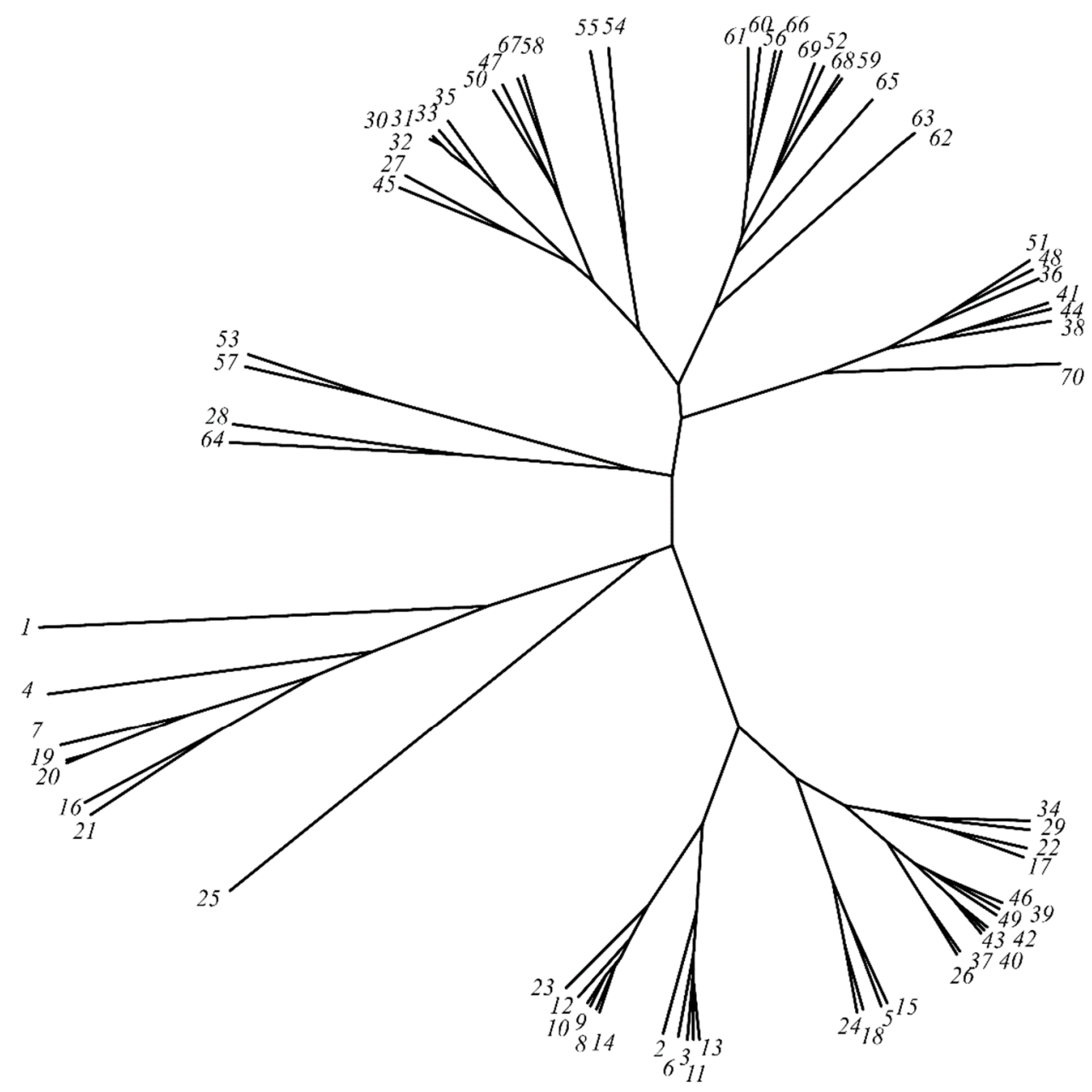

Figure 2. Unrooted cluster tree for different Hyssopus officinalis samples.

As $\beta$-pinene chemotype are classified two accessions (ssp. aristatus) with 19.3 and $24.7 \%$ of $\beta$-pinene [70]. As pinocamphone chemotype are classified accessions with ( 34.0 to 58.3$) \%$ of pinocamphone $[35,41,42,64-66,69]$. However, this is much more than the requirements of ISO 9841 Standard (8.0 to 25.0$) \%$.

Isopinocamphone chemotype is divided into several subgroups. Chemotype which contains between 33.6 and 44.7\% (satisfied requirements of ISO 9841 Standard), but only two samples $[4,56]$ contain enough pinocamphone $(14.1 \%)$, while another two $[9,63]$ contains pinocarvone and limonene, as well as $\beta$-pinene as significant compounds. In addition, isopinocamphone chemotype with (24.9 to 58.3 )\%, except two accessions with higher content of isopinocamphone [71,76] satisfies requirements of ISO 9841 Standard according to this compound. Samples between 16.3 and $21.1 \%$ of pinocamphone $[2,69,78,79]$ also satisfy the requirements of ISO 9841 Standard. However, in other samples, other major compounds are 1,8-cineole [75], $\beta$-pinene [74] and camphor [76]. Extremely rich isopinocamphone chemotype, with $(58.5$ to 72.8$) \%$ (accessions described by $[40,54,60]$ contain much more isopinocamphone than requirements of ISO 9841 Standard (25.0 to 45.0$) \%$.

The obtained data determine the position of the samples in the factor planes. The geometrically close points suggest the similitude of patterns represented by these points.

Three groups of mixed chemotypes pinocamphone + isopinocamphone $+\beta$-pinene mostly satisfies ISO 9841 Standard according to essential oil quality $[11,34,35,40,41,43,46$, 47,50-52,57,65].

Except for these four main chemotypes, there are pinocarvone [41,62,63,67,68,73,77], 1,8-cineole [53,58], limonene + methyl eugenol [70,72], linalool [7,9], thymol [55,59], and 
chemotypes with one accession: $\alpha$-pinene [49] and methyl acetate [61]. Correlation between these different $H$. officinalis samples is shown in Figure 3.

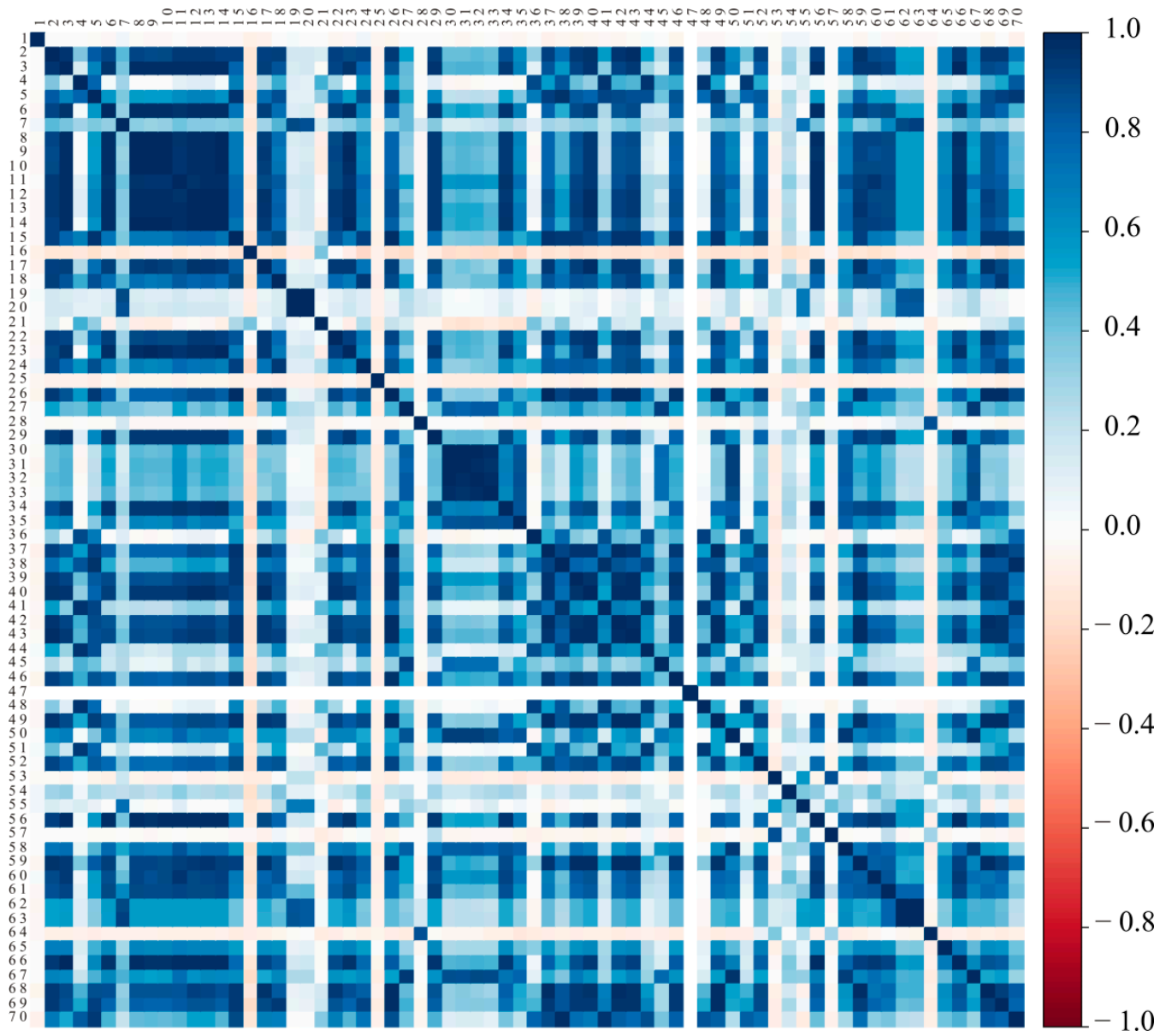

Figure 3. Correlation between different Hyssopus officinalis samples.

\subsection{Correlation Analysis}

The correlation analysis was performed to investigate the similarities in active compounds content of the different Hyssopus officinalis samples (1-70), and the results were visually displayed in Figure 3. It can be noticed from the figure that the darker blue colour of the squares, which shows the two samples relation, presents a stronger correlation between these samples, i.e., the more pronounced similarity in the active compound content. On the other hand, the lighter tone suggests a certain dissimilarity between samples. Therefore, if the colour tone is slighter, the correlation is lower.

\subsection{QSRR Analysis}

The PaDel-descriptor was employed to evaluate the molecular descriptors. They present many aspects of the investigated compounds and have been successfully used in QSRR investigation. Prior to the GA calculation, the factor analysis was performed to eliminate the descriptors with equal or almost equal values of the examined molecular structures. Only one of the correlated descriptors remained in the GA calculation. GA was used to select the most appropriate set of molecular descriptors while choosing the most relevant set of descriptors was realized using the evolution simulation [80,81]. The number of genes was equal to the number of the molecular descriptors obtained in the PaDel-descriptor. The population of the first generation in the GA calculation was selected randomly. The number of the genes was kept low during the GA calculation, to maintain a small subset of descriptors. The probability of generating zero for a gene was set at 
least $60 \%$. The operators used in the simulation were: crossover ( $90 \%$ probability) and mutation $(0.5 \%)$. A population size of 100 genes was chosen for GA, and evolution was allowed for over 50 generations. The evolution of the generations was stopped when $90 \%$ of the generations took the same fitness. As a result, the five most significant molecular descriptors selected by GA were: ATSC5e, AATSC1v, GATS3p, IC0 and MW.

Detailed explanations about the descriptors were found in the Handbook of Molecular Descriptors [82]. These descriptors encoded different aspects of the molecular structure and were applied to develop a QSRR model. Table 5 represents the correlation matrix among these descriptors.

Table 5. Correlation matrix of the molecular descriptors.

\begin{tabular}{ccccc}
\hline & AATSC1v & GATS3p & IC0 & MW \\
\hline ATSC5e & 0.213 & 0.205 & -0.062 & -0.102 \\
& $p=0.102$ & $p=0.115$ & $p=0.639$ & $p=0.436$ \\
AATSC1v & & 0.068 & 0.002 & 0.221 \\
& & $p=0.608$ & $p=0.989$ & $p=0.089$ \\
GATS3p & & 0.009 & -0.224 \\
& & & & $p=0.947$ \\
IC0 & & & -0.074 \\
& & & & 0.574 \\
\hline
\end{tabular}

ATSC5e-Centred Broto-Moreau autocorrelation-lag 5/weighted by Sanderson electronegativities; AATSC1vAverage centred Broto-Moreau autocorrelation-lag 1/weighted by van der Waals volumes; GATS3p-Geary autocorrelation-lag 3/weighted by polarizabilities; IC0 - Information content index (neighbourhood symmetry of 0-order); MW-Molecular weight.

The calibration and predictive capability of a QSRR model should be tested through model validation. The most widely used squared correlation coefficient $\left(r^{2}\right)$ can provide a reliable indication of the fitness of the model. Thus, it was employed to validate the calibration capability of a QSRR model.

To explore the nonlinear relationship between RIs and the selected descriptors, the ANN technique was used to build a retention time predictive model. The statistical results of the MLP 5-10-1 network are shown in Table 6.

Table 6. Goodness of fit of the ANN model.

\begin{tabular}{cccccccc}
\hline & $\chi^{2}$ & RMSE & MBE & MPE & SSE & AARD & $r^{2}$ \\
\hline$R I$ & 5853.304 & 75.867 & 16.527 & 5.212 & 328956.214 & 4367.929 & 0.910 \\
\hline
\end{tabular}

The quality of the model fit was tested in Table 4, in which the lower reduced chisquare $\left(\chi^{2}\right)$, mean bias error $(M B E)$, root mean square error (RMSE), mean percentage error $(M P E)$. Values show a better fit to the experimental results [83].

The predicted RIs are presented in Figure 4a to confirm the adequate prediction capabilities of the constructed ANN, by showing the relationship between the predicted and experimental retention values.

The highest deviation between retention time indices gained by experimental measurements and the ANN predicted retention time indices obtained were: 1,8-cineole; cis$\beta$-ocimene; trans- $\beta$-ocimene; terpinolene; cryptone; trans-2-hydroxy-pinocamphone; $\alpha$ copaene; $\delta$-elemene; $\beta$-bourbonene and $\alpha$-gurjunene.

Obtained results reveal the reliability of the ANN models for predicting the RIs of compounds in H. officinal is essential oil determined by GC-MS. 

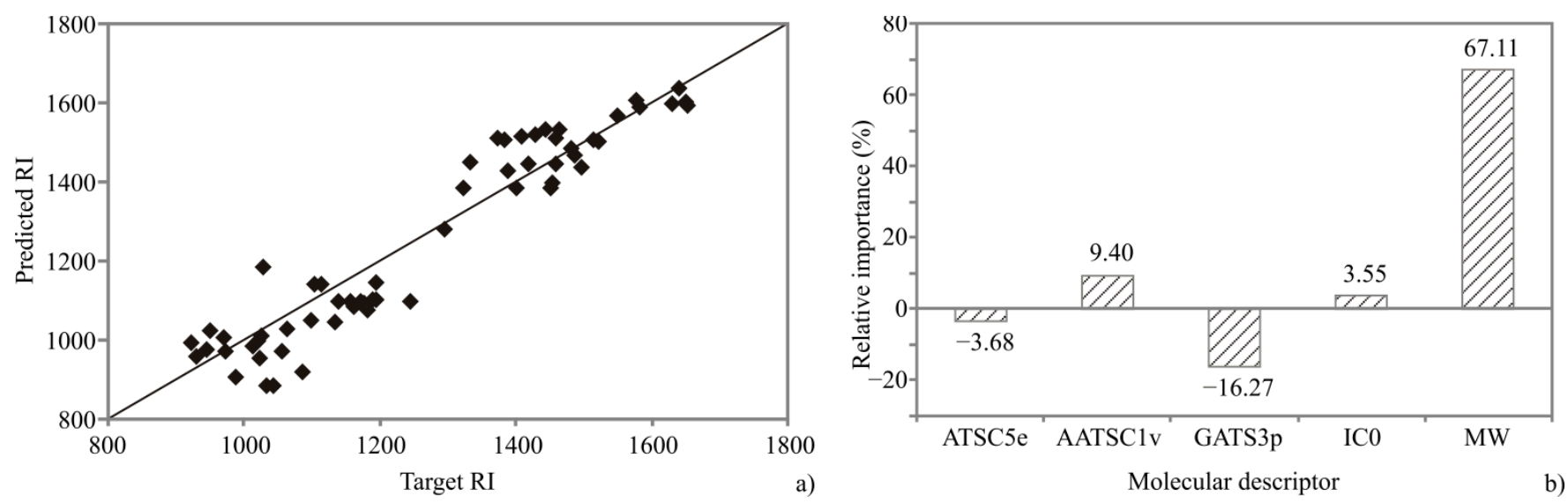

Figure 4. (a) Comparison of experimentally obtained RIs with ANN predicted values; (b) The relative importance of the molecular descriptors on RI, determined using Yoon interpretation method.

In this section, the influence of the five most important input variables, identified using the genetic algorithm on RI was studied. According to Figure 4b, MW was the most influential parameter with an approximately relative importance of $67.11 \%$, while the influence of GATS3p was $-16.27 \%$.

\section{Conclusions}

The results of the chemical composition analysis of Hyssopus officinalis ssp. officinalis grown during three growing years reviled isopinocamphone as the most abundant component, on which accumulation, the temperature had negative, while precipitation had a positive impact. The opposite effect was noticed on the content of pinocamphone and $\beta$-pinene. The chemical composition variations of $H$. officinalis oils can be associated with many factors; however, the most prominent is intraspecific diversity.

Obtained the excellent quality of $H$. officinalis essential oil indicated the possibility of producing this species in plantations in Serbia. Established the quantitative structure retention relationship model for predicting the retention times of chemical compounds in H. officinalis essential oil obtained by hydrodistillation and analysed by GC-MS using the genetic algorithm variable selection method and the artificial neural network model was shown as reliable and has great potential for practical use. The global sensitivity analysisYoon interpretation method identified molecular weight as the most influential parameter affecting the retention indices of chemical compounds in H. officinalis essential oil.

Author Contributions: Conceptualization, M.A. and V.S.; methodology, T.Z.; software, L.P.; validation, J.S.J., M.C. and M.I.; formal analysis, B.L. and J.S.J.; investigation, M.A. and M.C.; resources, A.M.J.; data curation, L.P.; writing—original draft preparation, B.L.; writing—review and editing, T.Z.; visualization, L.P.; supervision, A.M.J.; project administration, M.A.; funding acquisition, M.A., T.Z., A.M.J., V.S. and M.I. All authors have read and agreed to the published version of the manuscript.

Funding: This research was funded by the Ministry of Education, Science and Technological Development of the Republic of Serbia, grant number: 451-03-9/2021-14/200032 and Interreg-IPA CBC HUSRB7190374270059.

Institutional Review Board Statement: Not applicable.

Informed Consent Statement: Not applicable.

Data Availability Statement: Not applicable.

Conflicts of Interest: The authors declare no conflict of interest. 


\section{References}

1. Zawislak, G. Hyssop herb yield and quality depending on harvest term and plant spacing. Acta Sci. Pol. Hortorum Cultus 2011, 10, 331-342.

2. Aćimović, M.; Varga, A.; Cvetković, M.; Pezo, L.; Lončar, B.; Ignjatov, M.; Zeremski, T. Chemical composition of hyssop cv. "Domaći ljubičasti" essential oil and its antimicrobial activity. Ratarstvo Povrtarstvo 2021, 58, 23-30. [CrossRef]

3. Fathiazad, F.; Hamedeyazdan, S. A review on Hyssopus officinalis L.: Composition and biological activities. Afr. J. Pharm. Pharmacol. 2011, 5, 1959-1966. [CrossRef]

4. Moghtader, M. Comparative evaluation of the essential oil composition from the leaves and flowers of Hyssopus officinalis L. J. Hortic. For. 2014, 6, 1-5. [CrossRef]

5. Ahmadi, H.; Babalar, M.; Sarcheshmeh, M.A.A.; Morshedloo, M.R.; Shokrpour, M. Effects of exogenous application of citrulline on prolonged water stress damages in hyssop (Hyssopus officinalis L.): Antioxidant activity, biochemical indices, and essential oils profile. Food Chem. 2020, 333, 127433. [CrossRef] [PubMed]

6. Josifović, M. Flora SR Srbije; Srpska Akademija Nauke i Umetnosti: Beograd, Serbia, 1975; Volume 6.

7. Venditti, A.; Bianco, A.; Frezza, C.; Conti, F.; Bini, L.M.; Giuliani, C.; Bramucci, M.; Quassinti, L.; Damiano, S.; Lupidi, G.; et al. Essential oil composition, polar compounds, glandular trichomes and biological activity of Hyssopus officinalis subsp. aristatus (Godr.) Nyman from central Italy. Ind. Crops Prod. 2015, 77, 353-363. [CrossRef]

8. ISO 9841. Oil of Hyssopus officinalis L. ssp. officinalis; International Organisation for Standardization: Geneva, Switzerland, 2007.

9. Mazzanti, G.; Battinelli, L.; Salvatore, G. Antimicrobial properties of the linalol-rich essential oil of Hyssopus officinalis L. var decumbens (Lamiaceae). Flavour Fragr. J. 1998, 13, 289-294. [CrossRef]

10. Renzini, G.; Scazzocchio, F.; Lu, M.; Mazzanti, G.; Salvatore, G. Antibacterial and cytotoxic activity of Hyssopus officinalis L. oils. J. Essent. Oil Res. 1999, 11, 649-654. [CrossRef]

11. Kizil, S.; Hasimi, N.; Tolan, V.; Kilinc, E.; Karatas, H. Chemical composition, antimicrobial and antioxidant activities of hyssop (Hyssopus officinalis L.) essential oil. Not. Bot. Horti Agrobot. Cluj Napoca 2010, 38, 99-103.

12. Wolfender, J.L.; Martia, G.; Thomas, A.; Bertranda, S. Current approaches and challenges for the metabolite profiling of complex natural extracts. J. Chromatogr. A 2015, 1382, 136-164. [CrossRef]

13. Marrero-Ponce, Y.; Barigye, S.J.; Jorge-Rodriguez, M.E.; Tran-Thi-Thu, T. QSRR prediction of gas chromatography retention indices of essential oil components. Chem. Pap. 2017, 72, 57-69. [CrossRef]

14. Wu, L.; Gong, P.; Wu, Y.; Liao, K.; Shen, H.; Qi, Q.; Liu, H.; Wang, G.; Hao, H. An integral strategy toward the rapid identification of analogous nontarget compounds from complex mixtures. J. Chromatogr. A 2013, 1303, 39-47. [CrossRef] [PubMed]

15. Héberger, K. Quantitative structure-(chromatographic) retention relationships. J. Chromatogr. A 2007, 1158, 273-305. [CrossRef] [PubMed]

16. Kaliszan, R. QSRR: Quantitative Structure-(Chromatographic) Retention Relationships. Chem. Rev. 2007, 107, 3212-3246. [CrossRef] [PubMed]

17. Khezeli, T.; Daneshfar, A.; Sahraei, R. A green ultrasonic-assisted liquid-liquid microextraction based on deep eutectic solvent for the HPLC-UV determination of ferulic, caffeic and cinnamic acid from olive, almond, sesame and cinnamon oil. Talanta 2016, 150, 577-585. [CrossRef]

18. Tropsha, A.; Golbraikh, A. Predictive QSAR Modeling Workflow, Model Applicability Domains, and Virtual Screening. Curr. Pharm. Des. 2001, 3, 3494-3504. [CrossRef] [PubMed]

19. Zisi, C.; Sampsonidis, I.; Fasoula, S.; Papachristos, K.; Witting, M.; Gika, H.G.; Nikitas, P.; Pappa-Louisi, A. QSRR modeling for metabolite standards analyzed by two different chromatographic columns using multiple linear regression. Metabolites $2017,7,7$. [CrossRef]

20. ISO 10390:1994. Soil Quality—Determination of $\mathrm{pH}$; International Organization for Standardization: Geneva, Switzerland, 1994

21. ISO 10693:1995. Soil Quality_Determination of Carbonate Content-Volumetric Method; International Organization for Standardization: Geneva, Switzerland, 1995.

22. Berner, D.L.; Brown, J. Protein Nitrogen Combustion Method Collaborative Study I. Comparison with Smalley Total Kjeldahl Nitrogen and Combustion Results. J. Am. Oil Chem. Soc. 1994, 71, 1291-1293. [CrossRef]

23. Egner, H.; Riehm, H.; Domingo, W.R. Untersuchungenüber die chemische Boden analyse als Grundlage für die Beurteilung des Nahrstoff zustandes der Boden, II: Chemische Extractions metoden zu Phosphorund Kaliumbestimmung. K. Lantbr. Annaler. 1960, 26, 199-215.

24. Stanković, J.; Novaković, M.; Tešević, V.; Ćirić, A.; Soković, M.; Zdunić, G.; Dajić-Stevanović, Z.; Gođevac, D. HPTLC Bioautography-guided isolation of isogeranic acid as the main antibacterial constituent of Artemisia santonicum essential oil. J. Serb.Chem. Soc. 2019, 84, 1355-1365. [CrossRef]

25. Dong, J.; Cao, D.S.; Miao, H.Y.; Liu, S.; Deng, B.C.; Yun, Y.H.; Wang, N.N.; Lu, A.P.; Zeng, W.B.; Chen, A.F. ChemDes: An integrated web-based platform for molecular descriptor and fingerprint computation. J. Cheminform. 2015. [CrossRef] [PubMed]

26. Yap, C.W. PaDEL-descriptor: An open source software to calculate molecular descriptors and fingerprints. J. Comput. Chem. 2011, 32, 1446-1474. [CrossRef]

27. Goldberg, D.E. Genetic Algorithms in Search, Optimisation and Machine Learning; Addison-Wesley: Boston, MA, USA, 1989.

28. Tropsha, A. Best practices for QSAR model development, validation, and exploitation. Mol. Inform. 2010, 29, 476-488. [CrossRef] [PubMed] 
29. Aalizadeh, R.; Thomaidis, N.S.; Bletsou, A.A.; Gago-Ferrero, P. Quantitative structure-Retention relationship models to support nontarget high-resolution mass spectrometric screening of emerging contaminants in environmental samples. J. Chem. Inf. Model. 2016, 56, 1384-1398. [CrossRef] [PubMed]

30. Xu, Q.; Wei, C.; Liu, R.; Gu, S.; Xu, J. Quantitative structure-Property relationship study of $\beta$-cyclodextrin complexation free energies of organic compounds. Chemom. Intell. Lab. Syst. 2015, 146, 313-321. [CrossRef]

31. Kojic, P.; Omorjan, R. Predicting hydrodynamic parameters and volumetric gas-liquid mass transfer coefficient in an external-loop airlift reactor by support vector regression. Chem. Eng. Res. Des. 2018, 125, 398-407. [CrossRef]

32. Statistica 10 Software (StatSoft, Inc. STATISTICA, ver. 10, Data Analysis Software System). Available online: https://www. statsoft.de/en/home (accessed on 15 December 2018).

33. Yoon, Y.; Swales, G.; Margavio, T.M. A comparison of discriminant analysis versus Artificial Neural Networks. J. Oper Res. Soc. 2017, 44, 51-60. [CrossRef]

34. Acimovic, M.; Todosijević, M.; Varga, A.; Kiprovski, B.; Teševic, V.; Cabarkapa, I.; Sikora, V. Bioactivity of essential oils from cultivated winter savory, sage and hyssop. LekoviteSirovine 2019, 3, 11-17. [CrossRef]

35. Acimovic, M.; Stankovic, J.; Cvetkovic, M.; Kiprovski, B.; Marjanovic-Jeromela, A.; Rat, M.; Malencic, D. Essential oil analysis of different hyssop genotypes from IFVCNS medicinal plant collection garden. LetopisNaučnihRadova 2019, 43, 38-45.

36. Salvatore, G.; D’Andrea, A.; Nicoletti, M. A pinocamphone poor oil of Hyssopus officinalis L. var. decumbens from France (Banon). J. Essent. Oil. Res. 1998, 10, 563-567. [CrossRef]

37. Mutu, A.; Clapco, S.; Martea, R.; Port, A.; Gille, E.; Duca, M. Intraspecific genetic variability of Hyssopus officinalis L. J. Exp. Mol. Biol. 2014, 15, 1-8.

38. Saeedi, M.; Morteza-Semnani, K. Chemical composition and antimicrobial activity of essential oil of Hyssopus angustifolius M.B. J. Essent. Oil Bear. Plants 2009, 12, 111-119. [CrossRef]

39. Riabova, P.A.; Micic, D.; Bozovic, R.B.; Jovanovic, D.V.; Tomic, A.; Sovljanski, O.; Filip, S.; Tosti, T.; Ostojic, S.; Blagojevic, S.; et al. The chemical, biological and thermal characteristics and gastronomical perspectives of Laurus nobilis essential oil from different geographical origin. Ind. Crops Prod. 2020, 151, 112498. [CrossRef]

40. Nemeth-Zambori, E.; Rajhart, P.; Inotai, K. Effect of genotype and age on essential oil and total phenolics in hyssop (Hyssopus officinalis L.). J. Appl. Bot. Food Qual. 2017, 90, 25-30.

41. Kerrola, K.; Galambosi, B.; Kallio, H. Volatile components and odor intensity of four phenotypes of hyssop (Hyssopus officinalis L.). J. Agric. Food Chem. 1994, 42, 776-781. [CrossRef]

42. Kotyuk, L.A. Hyssop composition depending on age and plants development phases. Biotechnol. Acta 2015, 8, 55-63. [CrossRef]

43. Zawislak, G. Morphological characters of Hyssopus officinalis L. and chemical composition of its essential oil. Mod. Phytomorphol. 2013, 4, 93-95.

44. Karp, F.; Croteau, R. Hydroxylation of (-)- $\beta$-pinene and (-)- $\alpha$-pinene by a cytochrome P-450 system from hyssop (Hyssopus officinalis). In Secondary-Metabolite Biosynthesis and Metabolism; Petrovski, R.J., McCormick, S.P., Eds.; Plenum Press: New York, NY, USA, 1992.

45. Galambosi, B.; Svoboda, K.; Deans, S.; Hethelyi, E. Agronomical and phytochemical investigation of Hyssopus officinalis. Agric. Food Sci. 1993, 2, 293-302. [CrossRef]

46. Tavakoli, M.; Aghajani, Z. The effects of draught stress on the components of the essential oil of Hyssopus officinalis L. and determining the antioxidative properties of its water extracts. J. Appl. Environ. Biol. Sci. 2016, 6, 31-36.

47. Moro, A.; Zalacain, A.; de Mendoza, J.H.; Carmona, M. Effects of agronomic practices on volatile composition of Hyssopus officinalis L. essential oils. Molecules 2011, 16, 4131-4139. [CrossRef]

48. Hamida, N.B.; Martinez-Diaz, R.A.; Hela, M.; Msaada, K.; Ouerghi, Z.; Andres, M.F.; Gonzalez-Coloma, A. Effect of salinity on the antiparasitic activity of hyssop essential oil. J. Essent. Oil Res. 2020, 32, 69-78. [CrossRef]

49. Ogunwande, I.A.; Flamini, G.; Alese, O.O.; Cioni, P.L.; Ogundajo, A.L.; Setzer, W. A new chemical form of essential oil of Hyssopus officinalis L. (Lamiaceae) from Nigeria. Int. J. Biol Chem Sci. 2011, 5, 46-55. [CrossRef]

50. Glamočlija, J.M.; Soković, M.D.; Vukojević, J.B.; Milenković, I.M.; Brkić, D.D.; van Griensven, L.J.L.D. Antifungal activity of essential oil Hyssopus officinalis L. against mycopathogen Mycogone perniciosa (Mang). Proc. Nat. Sci. Matica Srpska Novi. Sad. 2005, 109, 123-128. [CrossRef]

51. Hristova, Y.; Wanner, J.; Jirovetz, L.; Stappen, I.; Iliev, I.; Gochev, V. Chemical composition and antifungal activity of essential oil of Hyssopus officinalis L. from Bulgaria against clinical isolates of Candida species. Biotechnol. Biotechnol. Equip. 2015, 29, 592-601. [CrossRef]

52. Baj, T.; Korona-Glowniak, I.; Kowalski, R.; Malm, A. Chemical composition and microbiological evaluation of essential oil from Hyssopus officinalis L. with white and pink flowers. Open Chem. 2018, 16, 317-323. [CrossRef]

53. Dzamic, A.; Soković, M.; Novakovic, M.; Jadranin, M.; Ristic, M.; Tesevic, V.; Marin, P. Composition, antifungal and antioxidant properties of Hyssopus officinalis L. subsp. pilifer (Pant.) Murb. Essential oil and deodorized extracts. Ind. Crops Prod. 2013, 51, 401-407. [CrossRef]

54. Gonceariuc, M.; Balmus, Z. Diversity of the essential oil content and chemical composition of Hyssopus officinalis L. genotype. Muzel Olteniei Craiova 2013, 29, 71-77.

55. Dehghanzadeh, N.; Ketabchi, S.; Alizsdeh, A. Essential oil composition and antibacterial activity of Hyssopus officinalis L. grown in Iran. Asian J. Exp. Boil. Sci. 2012, 3, 767-771. 
56. Mitic, V.; Dordevic, S. Essential oil composition of Hyssopus officinalis L. cultivated in Serbia. Facta Univ. 2000, 2, 105-108.

57. Said-Al Ahl, H.A.H.; Abbas, Z.K.; Sabra, A.S.; Tkachenko, K.G. Essential oil composition of Hyssopus officinalis L. cultivated in Egypt. Int. J. Plant. Sci. Ecol. 2015, 1, 49-53.

58. Zagorcheva, T.; Rusanov, K.; Rusanova, M.; Aneva, I.; Stancheva, I.; Atanassov, I. Genetic and flower volatile diversity in two natural populations of Hyssopus officinalis L. in Bulgaria. Biotechnol. Biotechnol. Equip. 2020, 34, 1265-1272. [CrossRef]

59. Li, H.T.; Zhao, N.N.; Yang, K.; Liu, Z.L.; Wang, Q. Chemical composition and toxicities of the essential oil derived from Hyssopus cuspidatus flowering aerial parts against Sitophilus zeamais and Heteroderaavenae. J. Med. Plants Res. 2013, 7, $343-348$.

60. Sharopov, F.S.; Kukaniev, M.A.; Thompson, R.M.; Satyal, P.; Setzer, W.N. Composition and antimicrobial activity of the essential oil of Hyssopus seravschanicus growing wild in Tajikistan. Der Pharma Chem. 2012, 4, 961-966.

61. Fathiazad, F.; Mazandarani, M.; Hamedeyazdan, S. Phytochemical analysis and antioxidant activity of Hyssopus officinalis L. from Iran. Adv. Pharm. Bull. 2011, 1, 63-67.

62. Kurkcuoglu, M.; Eser, S.A.; Baser, K.H.C. Composition of the essential oil of the Hyssopus officinalis L. susp. angustifolius (Bieb.) Arcangeli. Nat. Volatiles Essent. Oils 2016, 3, 15-19.

63. Bernotienè, G.; Butkienè, R. Essential oils of Hyssopus officinalis L. cultivated in East Lithuania. Chemija 2010, 21, 135-138.

64. Schulz, G.; Stahl-Biskup, E. Essential oil and glycosidic bound volatiles from leaves, stems, flowers and roots of Hyssopus officinalis L. (Lamiaceae). Flav. Fragr. J. 1991, 6, 69-73. [CrossRef]

65. Chalchat, J.C.; Adamovic, D.; Gorunovic, M.S. Composition of oils of three cultivated forms of Hyssopus officinalis endemic in Yugoslavia: F. albus Alef., f. cyaneus Alef. and f. ruber Mill. J. Essent. Oil Res. 2001, 13, 419-421. [CrossRef]

66. Garg, S.N.; Naqvi, A.A.; Singh, A.; Ram, G.; Kumar, S. Composition of essential oil from an annual crop of Hyssopus officinalis grown in Indian plains. Flav. Fragr. J. 1999, 14, 170-172. [CrossRef]

67. Ozer, H.; Sahin, F.; Kilic, H.; Gulluce, M. Essential oil composition of Hyssopus officinalis L. subsp. angustifolius (Bieb.) Arcangeli from Turkey. Flav. Frag. J. 2005, 20, 42-44.

68. Mohan, M.; Seth, R.; Singh, P.; Lohani, H.; Gupta, S. Composition of the volatiles of Hyssopus officinalis (L.) and Thymus serpyllum (L.) from Uttarakhand Himalaya. Natl. Acad. Sci. Lett. 2012. [CrossRef]

69. Fraternale, D.; Ricci, D.; Epifano, F.; Curini, M. Composition and antifungal activity of two essential oils of Hyssop (Hyssopus officinalis L.). J. Essent. Oil Res. 2004, 16, 617-622. [CrossRef]

70. Piccaglia, R.; Pace, L.; Tammaro, F. Characterization of essential oils from three Italian ecotypes of hyssop [Hyssopus officinalis L. subsp. aristatus (Godron) Briq]. J. Essent. Oil Bear. Plants 1999, 11, 693-699. [CrossRef]

71. Kizil, S.; Toncer, O.; Ipek, A.; Arslan, N.; Saglam, S.; Khawar, K.M. Blooming stages of Turkish hyssop (Hyssopus officinalis L.) affect essential oil composition. Acta Agric. Scand. B Soil Plant Sci. 2008, 58, 273-279.

72. Gorunovic, M.S.; Bogavac, P.M.; Chalchat, J.C.; Chabard, J.L. Essential oil of Hyssopus officinalis L., Lamiaceae of Montenegro origin. J. Essent. Oil Res. 1995, 7, 39-43. [CrossRef]

73. Hatipoglu, G.; Sokmen, M.; Bektas, E.; Daferera, D.; Sokmen, A.; Demir, E.; Sahin, H. Automated and standard extraction of antioxidant phenolic compounds of Hyssopus officinalis L. ssp. angustifolius. Ind. Crops Prod. 2013, 43, 427-433. [CrossRef]

74. Jahantigh, O.; Najafi, F.; Badi, H.N.; Khavari-Najed, A.; Sanjarian, F. Essential oil composition of hyssop (Hyssopus officinalis L.) under salt stress at flowering stage. J. Essent. Oil Res. 2016, 28, 458-464. [CrossRef]

75. Genova, E.; Petrova, S.; Dobos, A.; Mathe, I. Content and composition of essential oil of the Bulgarian populations of Hyssopus officinalis ssp. aristatus (Godr.) Briq. Med. Plant. Rep. Novi Sad 2000, 7, 10-15.

76. Pirbalouti, A.G.; Bajalan, I.; Malekpoor, F. Chemical composition and antioxidant activity of essential oils from inflorescens of two landraces of hyssop [Hyssopus officinalis L. subsp. angustifolius (Bieb.)] cultivated in Southwestern, Iran. J. Essent. Oil Bear Plants 2019, 22, 1074-1081. [CrossRef]

77. Stappen, I.; Wanner, J.; Tabanaca, N.; Wedge, D.; Ali, A.; Kaul, V.; Lal, B.; Gochev, V.; Schmidt, E.; Jirovetz, L. Chemical composition and biological activity of essential oils of Dracocephalum heterophyllum and Hyssopus officinalis from Western Himalaya. Nat. Prod. Commun. 2015, 10, 133-138. [CrossRef]

78. Mahboubi, M.; Haghi, G.; Kazempour, N. Antimicrobial activity and chemical composition of Hyssopus officinalis L. essential oil. J. Biol. Act. Prod. Nat. 2011, 1, 132-137.

79. Zawislak, G. Essential oil composition of Hyssopus officinalis L. grown in Poland. J. Essent. Oil Bear Plants 2016, 19, 699-705. [CrossRef]

80. Mohammadhosseini, M. Novel PSO-MLR Algorithm to Predict the Chromatographic Retention Behaviors of Natural Compounds. Anal. Chem. Lett. 2013, 3, 226-248. [CrossRef]

81. Nekoei, M.; Mohammadhosseini, M.; Pourbasheer, E. QSAR study of VEGFR-2 inhibitors by using genetic algorithm-multiple linear regressions (GA-MLR) and genetic algorithm-support vector machine (GA-SVM): A comparative approach. Med. Chem. Res. 2015, 24, 3037-3046. [CrossRef]

82. Todeschini, R.; Consonni, V. Handbook of Molecular Descriptors, Methods and Principles in Medicinal Chemistry; Wiley-VCH Verlag GmbH: Weinheim, Germany, 2000.

83. Arsenović, M.; Pezo, L.; Stanković, S.; Radojević, Z. Factor space differentiation of brick clays according to mineral content: Prediction of final brick product quality. Appl. Clay Sci. 2015, 115, 108-114. [CrossRef] 